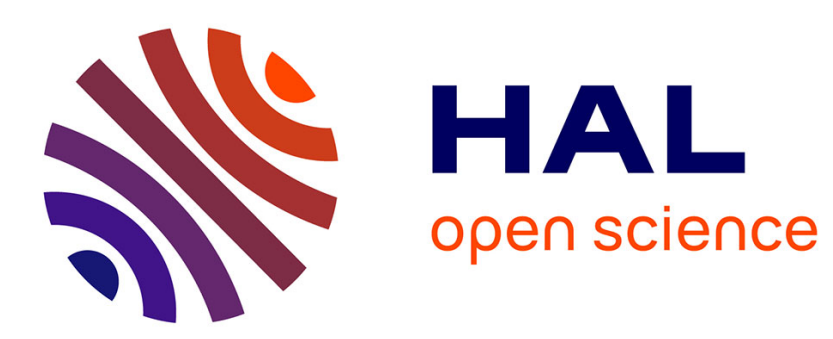

\title{
Amenability and non-uniform growth of some directed automorphism groups of a rooted tree
}

Jérémie Brieussel

\section{To cite this version:}

Jérémie Brieussel. Amenability and non-uniform growth of some directed automorphism groups of a rooted tree. Mathematische Zeitschrift, 2009, 263 (2), pp.265-293. hal-00797021

\section{HAL Id: hal-00797021 \\ https://hal.science/hal-00797021}

Submitted on 5 Mar 2013

HAL is a multi-disciplinary open access archive for the deposit and dissemination of scientific research documents, whether they are published or not. The documents may come from teaching and research institutions in France or abroad, or from public or private research centers.
L'archive ouverte pluridisciplinaire HAL, est destinée au dépôt et à la diffusion de documents scientifiques de niveau recherche, publiés ou non, émanant des établissements d'enseignement et de recherche français ou étrangers, des laboratoires publics ou privés. 


\title{
AMENABILITY AND NON UNIFORM GROWTH OF SOME DIRECTED AUTOMORPHISM GROUPS OF A ROOTED TREE
}

\author{
JÉRÉMIE BRIEUSSEL
}

\begin{abstract}
A result of amenability of some automorphism groups of a spherically homogeneous rooted tree of bounded valency is given. It is used to construct uncountably many amenable groups of non uniform exponential growth. Their Cayley graphs can be made arbitrary close to that of some groups of intermediate growth. Yet those groups are not in the class $S G$ of subexponentially amenable groups.
\end{abstract}

\section{INTRODUCTION}

Given a finitely generated group $\Gamma$ endowed with a generating set $S$ the growth function, $b_{\Gamma, S}(r)$ is defined as the number of group elements which are products of less than a given number $r$ of generators and their inverses. The growth of $\Gamma$ is qualified exponential when the exponential growth rate $h_{S}(\Gamma)=\lim \sqrt[r]{b_{\Gamma, S}(r)}$ strictly exceeds 1 for some, hence for all, generating set $S$. The growth is said intermediate if $h_{S}(\Gamma)=1$ and the growth function is not polynomial, that is when the group is not virtually nilpotent ([Gro1]). The growth is qualified uniform when the infimum of the exponential growth rates over all generating sets strictly exceeds 1, non uniform when exponential but: $\inf _{S} h_{S}(\Gamma)=1$.

The question of existence of groups of non uniform exponential growth was asked by Gromov in 1981 in the little green book [Gro2]. It has been shown that such groups do not occur in several classes such as hyperbolic groups (see [Kou]), linear groups (see [EMO]), elementary amenable groups (see [Osi2]). A pleasant exposition is given in $[\mathrm{dlH}]$. The first examples of such groups have been provided by Wilson in [Wil1] and [Wil2]. They contain free subgroups. Another example is due to Bartholdi in [Bar]. The main object of this paper is the following:

Theorem 1.1. There exist uncountably many pairwise non isomorphic amenable groups of non uniform exponential growth.

These groups will appear as subgroups of the group Aut $\left(T_{\bar{d}}\right)$ of automorphisms of a spherically homogeneous rooted tree, which is described. In Section 3 a subgroup of $\operatorname{Aut}\left(T_{\bar{d}}\right)$ is proved to be amenable in case of bounded valency of the tree. This Main Theorem 3.1 implies in particular that the group considered in [Bar] is amenable. Sections 4 and 5 are devoted to the proof of this Main Theorem. In Section 6, using specific generating sets of the alternate group of permutation, some groups of intermediate growth are introduced. These groups are proved to be dense in

Date: 12 February, 2008. 
the profinite group of alternate automorphism of the rooted tree. The groups of Theorem 1.1 are constructed in Section 7, using results of Wilson ([Wil2]). Some part of Wilson Theorem 7.1, namely the convergence to 1 of the exponential growth rates associated to different generating sets, is reinterpreted as a convergence of the Cayley graphs to Cayley graphs of the groups of intermediate growth introduced in the previous section. The last Section 8 deals with the question of subexponential amenability. The groups of non uniform exponential growth constructed are proved not to be in the class $S G$.

\section{Automorphisms of SPherically homogeneous Rooted trees}

2.1. Spherically homogeneous rooted tree. Given a sequence $\bar{d}=\left\{d_{j}\right\}_{j \geq 0}$ of integers $d_{j} \geq 2$, the associated spherically homogeneous rooted tree denoted $\bar{T}_{\bar{d}}$ is defined as follows: the vertices are indexed by all finite sequences $v=\left(i_{1} i_{2} \ldots i_{k}\right)$ with $i_{j}$ in $\left\{1,2, \ldots, d_{j-1}\right\}$, including the empty sequence $\emptyset$ called the root, and the edges link the pairs $\left\{\left(i_{1} i_{2} \ldots i_{k}\right),\left(i_{1} i_{2} \ldots i_{k} i_{k+1}\right)\right\}$. Note that the sequence $\bar{d}$ need not be infinite in which case the tree is finite.

The distance (each edge has length 1) from a vertex to the root is called the level of the vertex. The vertices of level $l(v)=n$ form the $n$th layer (or level) of cardinality $d_{0} d_{1} \ldots d_{n-1}$.

Each vertex $v$ of level $n$ gives rise to a spherically homogeneous rooted subtree $T_{v}$ when restricting to vertices of the form $\left(v i_{n} i_{n+1} \ldots i_{n+k}\right)$. The tree $T_{v}$ is isomorphic to the tree $T_{\sigma^{n} \bar{d}}$ associated to the sequence $\sigma^{n} \bar{d}=\left\{d_{j}\right\}_{j \geq n}$ (with $\sigma$ denoting the usual shift $\left.\sigma:\left(d_{0} d_{1} d_{2} \ldots\right) \mapsto\left(d_{1} d_{2} d_{3} \ldots\right)\right)$.

2.2. Automorphism group. An automorphism of $T_{\bar{d}}$ is a graph automorphism, that is a bijection of the set of vertices mapping edges to edges, which fixes the root. These properties imply that the layers are preserved, and an automorphism acts on a layer by permutation. The group of all such automorphisms will be denoted $\operatorname{Aut}\left(T_{\bar{d}}\right)$. Spherical homogeneity ensures that $\operatorname{Aut}\left(T_{\bar{d}}\right)$ and $\operatorname{Aut}\left(T_{\sigma \bar{d}}\right)$ are related by an isomorphism:

$$
\operatorname{Aut}\left(T_{\bar{d}}\right) \simeq \operatorname{Aut}\left(T_{\sigma \bar{d}}\right) \curlywedge S_{d_{0}} .
$$

Recall that $G$ 2 $S_{d} \simeq(G \times \cdots \times G) \rtimes S_{d}$ where $S_{d}$ (the group of permutation of the set $\{1,2, \ldots, d\})$ acts on the $d$ copies of $G$ by permutation. This identification will allow to write extensively $f=\left(f_{1}, f_{2}, \ldots, f_{d_{0}}\right) \sigma$ with $f$ in $\operatorname{Aut}\left(T_{\bar{d}}\right)$, the $f_{i}$ in $\operatorname{Aut}\left(T_{\sigma \bar{d}}\right)$ and $\sigma$ in $S_{d_{0}}$. The product rule is $f g=\left(f_{1}, f_{2}, \ldots, f_{d_{0}}\right) \sigma\left(g_{1}, g_{2}, \ldots, g_{d_{0}}\right) \tau=$ $\left(f_{1} g_{\sigma(1)}, \ldots, f_{d_{0}} g_{\sigma\left(d_{0}\right)}\right) \sigma \tau$. In particular, there is a projection $p: \operatorname{Aut}\left(T_{\bar{d}}\right) \rightarrow S_{d_{0}}$ called restriction to the first level. The kernel of this projection is called the stabilizer of the first level, denoted $S t_{1}\left(A u t\left(T_{\bar{d}}\right)\right)$, easily checked to be isomorphic to the direct product $\operatorname{Aut}\left(T_{\sigma \bar{d}}\right) \times \cdots \times \operatorname{Aut}\left(T_{\sigma \bar{d}}\right)$ with $d_{0}$ factors.

More generally for each integer $n$, there is an isomorphism:

$$
\operatorname{Aut}\left(T_{\bar{d}}\right) \simeq \operatorname{Aut}\left(T_{\sigma^{n} \bar{d}}\right)<\operatorname{Aut}\left(T_{d_{0} \ldots d_{n-1}}\right),
$$

where $\operatorname{Aut}\left(T_{d_{0} \ldots d_{n-1}}\right)$ acts by permutation on $d_{0} \ldots d_{n-1}$ copies of $A u t\left(T_{\sigma^{n} \bar{d}}\right)$ the way it acts on the set of leaves $\partial T_{d_{0} \ldots d_{n-1}}$ (the boundary) of the finite tree $T_{d_{0} \ldots d_{n-1}}$. There 


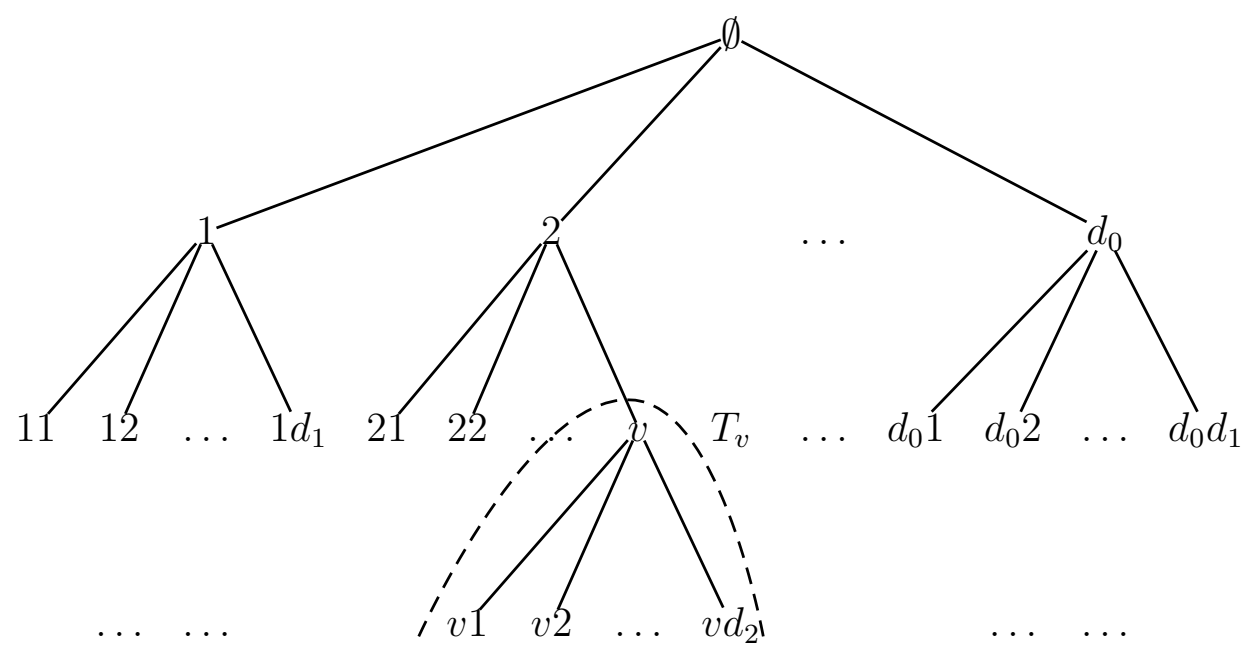

FigURE 1. Spherically homogeneous rooted tree, subtree.

is also a projection $p_{n}: \operatorname{Aut}\left(T_{\bar{d}}\right) \rightarrow \operatorname{Aut}\left(T_{d_{0} \ldots d_{n-1}}\right)$, the kernel of which constitutes the stabilizer $S t_{n}\left(\operatorname{Aut}\left(T_{\bar{d}}\right)\right)$ of the $n$th level. This is a normal subgroup of $\operatorname{Aut}\left(T_{\bar{d}}\right)$ isomorphic to the direct product $S t_{n}\left(\operatorname{Aut}\left(T_{\bar{d}}\right)\right) \simeq \operatorname{Aut}\left(T_{\sigma^{n} \bar{d}}\right) \times \cdots \times \operatorname{Aut}\left(T_{\sigma^{n} \bar{d}}\right)$, the elements of which will occasionally be written $g=\left(g_{1 \ldots 1}, \ldots, g_{d_{0} \ldots d_{n-1}}\right)_{n}$.

The full group of automorphism can be viewed as a profinite group via:

$$
\operatorname{Aut}\left(T_{\bar{d}}\right)=\lim _{n \rightarrow \infty} \operatorname{Aut}\left(T_{d_{0} \ldots d_{n-1}}\right)=\lim _{n \rightarrow \infty}\left(S_{d_{n-1}} \prec S_{d_{n-2}} \prec \cdots \succ S_{d_{0}}\right) \text {. }
$$

A basis of open sets for the profinite topology associated is $\left\{S t_{n}\left(A u t\left(T_{\bar{d}}\right)\right)\right\}_{n \geq 0}$. This topology can also be defined as associated to any of the following metrics $\delta_{\bar{\lambda}}$ on $\operatorname{Aut}\left(T_{\bar{d}}\right)$. Given a decreasing sequence $\bar{\lambda}=\left\{\lambda_{n}\right\}_{n \geq 0}$ of positive numbers tending to zero, set:

$$
\delta_{\bar{\lambda}}(g, h)=\inf \left\{\lambda_{n} \mid g(v)=h(v) \quad \text { for all vertices } v \text { of level } \leq n\right\} .
$$

A nice description of automorphisms of a rooted tree is to draw portraits. A portrait is a function $g$ from the set of all vertices $v$ of the tree $T_{\bar{d}}$ taking permutation values $g(v) \in S_{d_{l(v)}}$. A portrait gives rise to a unique automorphism via the formula:

$$
g\left(i_{1} i_{2} i_{3} \ldots i_{k}\right)=\left(g(\emptyset) i_{1}\right)\left(g\left(i_{1}\right) i_{2}\right)\left(g\left(i_{1} i_{2}\right) i_{3}\right) \ldots\left(g\left(i_{1} \ldots i_{k-1}\right) i_{k}\right) .
$$

Conversely, every automorphism has a unique portrait. The metrics $\delta_{\bar{\lambda}}$ are such that two automorphisms are $n$-close if their portraits coincide on the $n$ first layers.

An automorphism is said to be even (or alternate) if all the permutations $g(v) \in$ $S_{d_{l(v)}}$ involved in the portrait are alternate permutations $g(v) \in \mathcal{A}_{d_{l(v)}}$. The group of alternate automorphisms will be denoted $A u t^{e}\left(T_{\bar{d}}\right)$. It satisfies:

$$
\operatorname{Aut}^{e}\left(T_{\bar{d}}\right)=\lim _{n \rightarrow \infty} A u t^{e}\left(T_{d_{0} \ldots d_{n-1}}\right)=\lim _{n \rightarrow \infty} \mathcal{A}_{d_{n-1}} \prec \mathcal{A}_{d_{n-2}} \prec \cdots \curlywedge \mathcal{A}_{d_{0}},
$$

the profinite topology, the distances associated and the stabilizers of levels are defined in the same way as for the full automorphism group. Note that if $T_{2}$ is a 2-regular rooted tree, then $A u t^{e}\left(T_{2}\right)$ is the trivial group. 


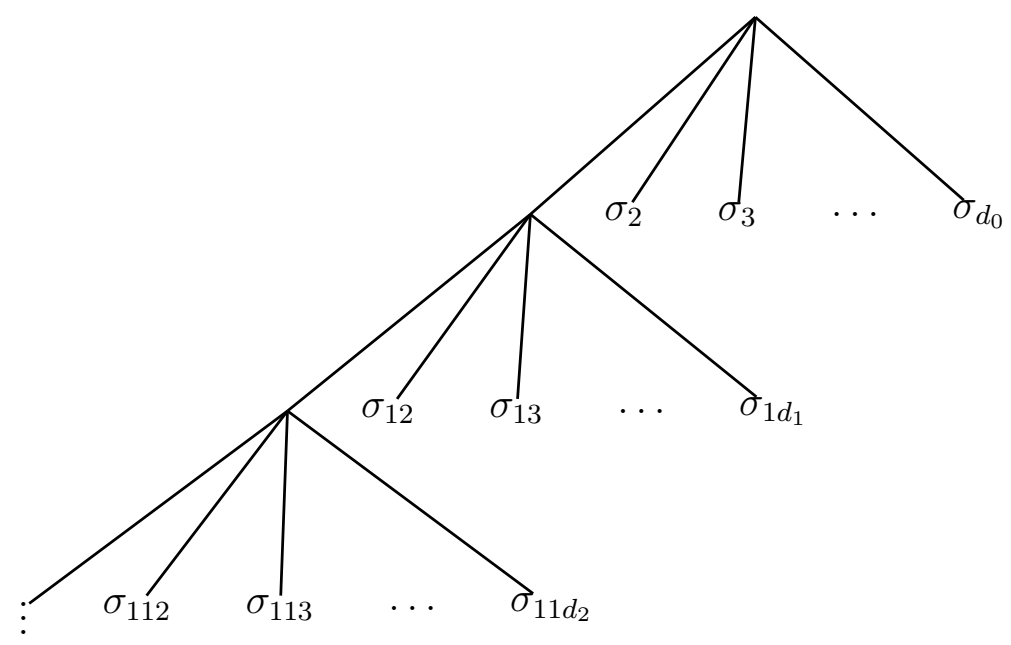

Figure 2. The $\iota$-action of $\bar{H}$.

2.3. Directed automorphism subgroups. This paper focuses on specific subgroups of $A u t\left(T_{\bar{d}}\right)$, those directed by a given infinite geodesic of the tree $T_{\bar{d}}$ starting from the root. Such a geodesic can always be chosen to be that passing at all vertices indexed by $11 \ldots 1$ (the leftmost geodesic in the illustrations). First introduce actions of some permutation groups on $T_{\bar{d}}$. The group $S_{d_{0}}$ acts on the rooted tree by permuting the subtrees of the first layer:

$$
\iota_{0}: S_{d_{0}} \hookrightarrow \operatorname{Aut}\left(T_{\bar{d}}\right) .
$$

More precisely, $\iota_{0}$ is defined by $\iota_{0}(\sigma)\left(i_{1} i_{2} \ldots i_{k}\right)=\sigma\left(i_{1}\right) i_{2} \ldots i_{k}$. For simplicity of notation, we will identify $\sigma=\iota_{0}(\sigma)=\left(i d_{T_{\sigma \bar{d}}}, \ldots, i d_{T_{\sigma \bar{d}}}\right) \sigma$ and call those rooted automorphisms (their portrait is trivial outside of the root).

The infinite direct product $\bar{H}=S_{d_{1}} \times \cdots \times S_{d_{1}} \times S_{d_{2}} \times \cdots \times S_{d_{2}} \times \ldots$ of permutation groups where $S_{d_{k}}$ appears $d_{k-1}-1$ times also acts in a canonical way (once a geodesic is chosen) on the rooted tree $T_{\bar{d}}$ :

$$
\iota: \bar{H} \hookrightarrow \operatorname{Aut}\left(T_{\bar{d}}\right) .
$$

Indeed, consider the vertices $1_{k} i=1 \ldots 1 i$ with $k$ ones and $i$ in $\left\{2, \ldots, d_{k}\right\}$. They form the set $P$ of vertices at distance exactly 1 of the leftmost geodesic $111 \ldots$ Each permutation group $S_{d_{k}}$ acts on a subtree $T_{1_{k} i}$ via the above homomorphism $\iota_{0}$ (corresponding to the rooted tree $T_{\sigma^{k}} \bar{d}$ ). More precisely, the action is recursively defined through the wreath product by:

$$
\iota\left(\sigma_{2}, \ldots, \sigma_{d_{0}}, \sigma_{12}, \ldots, \sigma_{1 d_{1}}, \ldots\right)=\left(\iota^{\prime}\left(\sigma_{12}, \ldots, \sigma_{1 d_{1}}, \ldots\right), \sigma_{2}, \ldots, \sigma_{d_{0}}\right),
$$

where $\iota^{\prime}\left(\sigma_{12}, \ldots, \sigma_{1 d_{1}}, \ldots\right)$ represents the action of the restriction $\bar{H} \rightarrow \bar{H}_{1}$ via:

$$
\iota^{\prime}: \bar{H}_{1}=S_{d_{2}}^{d_{1}-1} \times S_{d_{3}}^{d_{2}-1} \times \ldots \hookrightarrow \operatorname{Aut}\left(T_{1}\right) \simeq \operatorname{Aut}\left(T_{\sigma \bar{d}}\right)
$$

The geometry of the set $P$ ensures that the action of different factors commute, thus $\iota$ is a well defined injection. This is best understood by Figure 2, showing the portrait is non trivial only on $P$. The automorphisms obtained in $\iota(\bar{H})$ are said to be directed by the geodesic $111 \ldots$ 
Given a subgroup $A$ of $S_{d_{0}}$ and a subgroup $H$ of $\bar{H}$, denote by $G(A, H)$ the subgroup of $A u t\left(T_{\bar{d}}\right)$ generated by $\iota_{0}(A)$ and $\iota(H)$. Such a group will be called a directed group of automorphisms. Note that the group $H$ might not be countable as $\bar{H}$ is not. The group $G\left(S_{d_{0}}, \bar{H}\right)$ will be called full group of directed automorphisms. Note that the isomorphism (2.1) induces an isomorphism:

$$
G\left(S_{d_{0}}, \bar{H}\right) \simeq G\left(S_{d_{1}}, \bar{H}_{1}\right) \curlywedge S_{d_{0}} .
$$

The class of groups of the form $G(A, H)$ has been considered in [Gri2]. It gathers many famous examples such as the family of Aleshin-Grigorchuk groups known to be torsion (see [Ale1]) and of intermediate growth (see [Gri1]). Other interesting examples are some groups of non uniform growth constructed by Wilson ([Wil1], [Wil2]) and Bartholdi ([Bar]), to which Section 7 is devoted.

\section{The MAIN THEOREM}

In this section the Main theorem on full directed automorphism groups is stated and its proof is reduced to the proof of the a priori weaker Theorem 3.2.

Theorem 3.1 (Main Theorem). Let $\bar{d}=\left(d_{i}\right)_{i \geq 0}$ be a sequence of integers $d_{i} \geq 2$, let $S_{d_{0}}, \bar{H}$ and $G\left(S_{d_{0}}, \bar{H}\right)$ be the full directed subgroup of Aut $\left(T_{\bar{d}}\right)$, then:

1) if the sequence $\bar{d}$ is bounded, the group $G\left(S_{d_{0}}, \bar{H}\right)$ is amenable.

2) if the sequence $\bar{d}$ is unbounded, the group $G\left(S_{d_{0}}, \bar{H}\right)$ contains a free group $\mathbb{F}_{2}$ on two generators.

The proof of part 1) of the Main Theorem 3.1 reduces to proving the following, which will be the object of Sections 4 and 5 .

Theorem 3.2. Let $\bar{d}=\left(d_{i}\right)_{i \geq 0}$ be a bounded sequence of integers $2 \leq d_{i} \leq D$, let $H<\bar{H}$ be a finite saturated subgroup, then the directed subgroup $G\left(S_{d_{0}}, H\right)$ of $\operatorname{Aut}\left(T_{\bar{d}}\right)$ is amenable.

Proof of part 2). The second part of the Main Theorem is an immediate consequence of the following lemma stated in [Wil2] (see also [TW]).

Lemma 3.3 ([Wil2]). Let $F$ be the free product of two non-trivial finite groups which are not both of order 2 , and $\mathcal{S}$ be any infinite subset of $\mathbb{N}$. Then the alternate permutation group $\mathcal{A}_{d}$ is a homomorphic image of $F$ for all sufficiently large $d$ and the intersection of the kernels of all epimorphisms from $F$ to groups $\mathcal{A}_{d}$ with $d \in \mathcal{S}$ is the trivial subgroup.

This implies that if $\bar{d}$ is unbounded then the group $\bar{H}$ already contains a free group $\mathbb{F}_{2}$ on two generators. Indeed, let $F=\mathbb{Z} / 2 \mathbb{Z} \star \mathbb{Z} / 3 \mathbb{Z}$ the free group generated by elements $x$ of order 2 and $y$ of order 3 . Let $D$ be such that there is an onto homomorphism $\varphi_{d}: F \rightarrow \mathcal{A}_{d}$ when $d \geq D$. Define:

$$
\begin{gathered}
h_{1}=\left(\varphi_{d_{1}}(x), \ldots, \varphi_{d_{1}}(x), \varphi_{d_{2}}(x), \ldots, \varphi_{d_{2}}(x), \ldots\right) \in \bar{H} \\
h_{2}=\left(\varphi_{d_{1}}(y), \ldots, \varphi_{d_{1}}(y), \varphi_{d_{2}}(y), \ldots, \varphi_{d_{2}}(y), \ldots\right) \in \bar{H}
\end{gathered}
$$


where $\varphi_{d}(x)=\varphi_{d}(y)=1 \in \mathcal{A}_{d} \subset S_{d}$ if $d<D$. Then Lemma 3.3 ensures that the subgroup $\left\langle h_{1}, h_{2}\right\rangle<\bar{H}$ is isomorphic to $F$ which contains $\mathbb{F}_{2}$ as a subgroup of finite index.

If the sequence $\bar{d}$ is bounded then the properties of the group $\bar{H}$ are much different.

Fact 3.4. Let $\bar{H}=T_{1} \times T_{2} \times \ldots$ where the groups $T_{i}$ belong to a finite family $\mathcal{F}=\left\{F_{1}, \ldots F_{D}\right\}$ of finite groups, then every finitely generated subgroup $H^{\prime}$ of $\bar{H}$ is finite.

Proof. Let $h_{1}, \ldots, h_{k}$ be generators of $H^{\prime}$, they are of the form $h_{j}=\left(h_{j}^{1}, h_{j}^{2}, \ldots\right)$ with $h_{j}^{i} \in T_{i}$. There are at most $M=D \cdot\left(\max \left\{\# F_{i}\right\}\right)^{k}$ different $k+1$-tuples $\left(h_{1}^{i}, h_{2}^{i}, \ldots, h_{k}^{i}, F_{i}\right)$. Let $I$ be a subset of $\mathbb{N}$ of size less than $M$ such that all different $(k+1)$-tuples appear when $i$ describes $I$. Then the projection $\pi_{I}: \bar{H} \rightarrow \times_{i \in I} T_{i}$ is injective, so that $H^{\prime}$ is finite.

Definition 3.5. A finite subgroup $H$ of the group $\bar{H}=T_{1} \times T_{2} \times \ldots$ where the $T_{i}$ belong to a finite family $\mathcal{F}$ of finite group is said to be saturated if the equidistributed probability measure $q_{H}$ on $H$ projects on each coordinate $i$ to the equidistributed probability measure $q_{T_{i}}$ on $T_{i}$, that is if $h=\left(h_{1}, h_{2}, \ldots\right) \in H$ then $q_{H}\left(h_{i}=t\right)=$ $q_{T_{i}}(t)=\frac{1}{\# T_{i}}$.

Fact 3.6. Every finite subgroup $H^{\prime}$ of $\bar{H}$ is included in a finite saturated group $H$.

Proof. With the above notations set for each $i$ in $I$ :

$$
J_{i}=\left\{j \in \mathbb{N} \mid\left(h_{1}^{j}, h_{2}^{j}, \ldots, h_{k}^{j}, F_{j}\right)=\left(h_{1}^{i}, h_{2}^{i}, \ldots, h_{k}^{i}, F_{i}\right)\right\} .
$$

There is a diagonal embedding $T_{i} \rightarrow \times_{j \in J_{i}} T_{j}$ and as $\cup_{i \in I} J_{i}=\mathbb{N}$ we get a diagonal injection:

$$
\times_{i \in I} T_{i} \hookrightarrow \bar{H}
$$

the image $H$ of which contains $H^{\prime}$ and is saturated by construction, knowing a finite direct product is always saturated.

Proof that Theorem 3.2 implies the Main Theorem. To prove the group $G\left(S_{d_{0}}, \bar{H}\right)$ is amenable, it is sufficient to prove amenability for every finitely generated subgroup $G_{f}$ (Theorem 1.2.7. in [Gre]), which reduces, assuming Theorem 3.2, to show that $G_{f}$ is included in some $G\left(S_{d_{0}}, H\right)$ for $H$ finite saturated. Indeed, let $s_{1}, \ldots, s_{k}$ be generators of $G_{f}$, each $s_{j}$ is of the form $s_{j}=a_{j}^{1} h_{j}^{2} a_{j}^{3} \ldots h_{j}^{n_{j}}$, with $a_{j}^{i} \in S_{d_{0}}$ and $\left\langle\left(h_{j}^{i}\right)_{i, j}\right\rangle<\bar{H}$ finitely generate a subgroup $H^{\prime}$ which is included in some finite saturated subgroup $H$ by Facts 3.4 and 3.6.

\section{Scheme of the Proof of Theorem 3.2}

This section is devoted to the scheme of the proof of Theorem 3.2 which implies the Main Theorem 3.1. The details are given in Section 5. Groups of the form $G\left(S_{d_{0}}, H\right)$ share similarities with the Basilica group defined by a three state automaton introduced by Grigorchuk and Zuk in [GZ]. The Basilica group was shown to be amenable by Bartholdi and Virag (see [BV]) using selfsimilarity of some random walks. This method, called the "Münchhausen trick", has been used to show 
amenability of a few other groups (see [Kai] and [Muc]). We proceed with the same methods, using Kesten's criterion on symmetric random walks.

As $H$ is a finite saturated subgroup of $\bar{H}=S_{d_{1}}^{d_{0}-1} \times S_{d_{2}}^{d_{1}-1} \times \ldots$, let us denote $H_{k}$ its restriction to $\bar{H}_{k}=S_{d_{k+1}}^{d_{k}-1} \times S_{d_{k+2}}^{d_{k+1}-1} \times \ldots$ which is also a finite saturated subgroup and it follows from (2.5) that $G\left(S_{d_{0}}, H\right) \hookrightarrow G\left(S_{d_{1}}, H_{1}\right) \curlywedge S_{d_{0}}$, and more generally the group $G\left(S_{d_{k}}, H_{k}\right)$ is a directed subgroup of $A u t\left(T_{\sigma^{k}}\right)$ satisfying the crucial:

$$
G\left(S_{d_{k}}, H_{k}\right) \hookrightarrow G\left(S_{d_{k+1}}, H_{k+1}\right) \text { 乙 } S_{d_{k}} .
$$

The word metric does not behave appropriately enough through this wreath product embedding, rather use:

Proposition 4.1 (A fractal family of pseudo norms of exponential growth). There exists a family of pseudo norms $\nu^{k}$ on $G\left(S_{d_{k}}, H_{k}\right)$ (which means symmetric positive functions $\nu^{k}: G\left(S_{d_{k}}, H_{k}\right) \rightarrow \mathbb{R}^{+}$satisfying the triangle inequality) such that:

a) if $g$ belongs to $G\left(S_{d_{k}}, H_{k}\right)$ and has image $g=\left(g_{1}, \ldots, g_{d_{k}}\right) \sigma$ in $G\left(S_{d_{k+1}}, H_{k+1}\right) \curlywedge S_{d_{k}}$, then $\nu^{k}(g) \leq \nu^{k+1}\left(g_{1}\right)+\cdots+\nu^{k+1}\left(g_{d_{k}}\right)$, and

b) if $B_{\nu^{k}}(r)=\left\{g \in G\left(S_{d_{k}}, H_{k}\right) \mid \nu^{k}(g) \leq r\right\}$, then $\# B_{\nu^{k}}(r) \leq C^{r}$ where $C$ is a constant depending only on the bound $D$ on the valencies of the tree and the size of the finite group $H$ (which contains $H_{k}$ for every $k$ ).

Let $p$ denote the symmetric probability measure on the finite generating set $S_{d_{0}} \cup$ $H$ of $G\left(S_{d_{0}}, H\right)$ defined by $p(a)=\frac{1}{2 \# S_{d_{0}}}$ for $a \in S_{d_{0}}$ and $p(h)=\frac{1}{2 \# H}$ for $h \in H$. The random walk associated is $Z_{N}=s_{1} \ldots s_{N}$ where the $s_{i}$ are independent random variables identically $p$-distributed. The set of random sequences $\left(Z_{N}\right)_{N \in \mathbb{N}}$ is endowed with the product measure (defined on the sigma algebra generated by cylinders) $P=p^{\otimes \infty}$. The drift of this random walk with respect to the pseudo norm $\nu=\nu^{0}$ vanishes:

Proposition 4.2. The random walk $\left(Z_{N}\right)$ satisfies:

$$
\frac{\nu\left(Z_{N}\right)}{N} \underset{N \rightarrow+\infty}{\longrightarrow} 0, \quad P \text { a.s. }
$$

To prove this proposition, another (non symmetric) random walk is usefull. Let us define $Y_{n}=t_{0} t_{1} t_{2} \ldots t_{n}$ where $t_{2 i}$ are random variables equidistributed on $S_{d_{0}}$ and $t_{2 i+1}$ are equidistributed on $H$ and all the $t_{i}$ are independent. Denote $Q=$ $\left(q_{S_{d_{0}}} \otimes q_{H}\right)^{\otimes \infty}$ the associated measure on the set of sequences $\left(Y_{n}\right)_{n \in \mathbb{N}}$ (with respect to the cylindrical sigma algebra), then:

Proposition 4.3. The random walk $\left(Y_{n}\right)$ satisfies:

$$
\frac{\nu\left(Y_{n}\right)}{n} \underset{n \rightarrow+\infty}{\longrightarrow} 0, \quad Q \text { a.s.. }
$$

The key argument to prove Proposition 4.3 is the next Lemma 4.6 together with Proposition $4.1 \mathrm{a}$ ).

Remark 4.4 (On the dependence on $t_{0}$ ). The pseudo norm $\nu=\nu^{0}$ satisfies $\nu(a h)=$ $\nu(h)$ for every $a$ in $S_{d_{0}}$ and $h$ in $H$ (Proposition $5.2(2)$ ), which ensures $\nu\left(Y_{n}\right)=$ 
$\nu\left(t_{0}^{-1} Y_{n}\right)=\nu\left(t_{1} t_{2} \ldots t_{n}\right)$, showing that $\nu\left(Y_{n}\right)$ is independent of $t_{0}$. This will be of importance and justifies the:

Definition 4.5. Two random variables $U$ and $V$ on $G\left(S_{d_{0}}, H\right)$ are said $\nu$-equivalent if $\nu(U)$ and $\nu(V)$ have the same distribution law on $\mathbb{N}$, which will be denoted:

$$
U \sim_{\nu \text {-law }} V .
$$

Consider the random walk $\left(Y_{n}\right)_{n \in \mathbb{N}}$ and its image in the wreath product of the form $Y_{n}=\left(Y_{n}^{1}, \ldots, Y_{n}^{d_{0}}\right) \sigma_{n}$ where $\sigma_{n}$ is a random variable in $S_{d_{0}}$ and the coordinates $Y_{n}^{t}$ for $t \in\left\{1, \ldots, d_{0}\right\}$ are random variables in $G\left(S_{d_{1}}, H_{1}\right)$. The point is that $\left(Y_{n}^{t}\right)_{n}$ follows the law of the similarly defined random walk $\left(Y_{m}^{\prime}\right)_{m \in \mathbb{N}}$ on $G\left(S_{d_{1}}, H_{1}\right)$ (which is taking independent equidistributed increments alternatively in $S_{d_{1}}$ and $\left.H_{1}\right)$, but at a slower speed. More precisely:

Lemma 4.6 (Similarity of the random walks $\left(Y_{n}\right)$ and $\left(Y_{m}^{\prime}\right)$ ). Let $\left(Y_{n}\right)_{n \in \mathbb{N}}$ the random walk defined above and $Y_{n}=\left(Y_{n}^{1}, \ldots, Y_{n}^{d_{0}}\right) \sigma_{n}$ its image in the wreath product. For each coordinate $\left(Y_{n}^{t}\right)_{n}$ the sequence $\left(Y_{n}\right)_{n}$ defines a sequence of random integers $\left(m_{t}(n)\right)_{n}$ and a random sequence $\left(\varepsilon_{t}(n)\right)_{n}$ taking values in $\{0,1\}$ such that:

(1) For every integer $n$ the values of $m_{t}(n)$ and $\varepsilon_{t}(n)$ depend only on $\left(Y_{n^{\prime}}\right)_{n^{\prime} \leq n}$.

(2) For every integer $n$ the coordinate $Y_{n}^{t}$ belonging to $G\left(S_{d_{1}}, H_{1}\right)$ has the same $\nu^{1}$-distribution law as the random variable $Y_{m_{t}(n)+\varepsilon_{t}(n)}^{\prime}$. More precisely the conditional law:

$$
\left(Y_{n}^{t} \mid m_{t}(n), \varepsilon_{t}(n)\right) \sim_{\nu^{1-l a w}} Y_{m_{t}(n)+\varepsilon_{t}(n)}^{\prime}
$$

(3) The random sequence $\left(m_{t}(n)\right)_{n}$ satisfies:

$$
m_{t}(n) \sim_{n \rightarrow+\infty}\left(\frac{d_{0}-1}{d_{0}}\right) \frac{n}{d_{0}}, \quad Q \text { a.s.. }
$$

Propositions 4.1 and 4.2 are sufficient to apply the:

Theorem 4.7 (Kesten criterion of amenability [Kes]). Let $\Gamma$ be a finitely generated group and $\left(Z_{N}\right)$ a symmetric random walk on $\Gamma$. The group $\Gamma$ is amenable if and only if the sequence $\left(P\left(Z_{2 N}=i d_{\Gamma}\right)\right)_{N}$ does not decay exponentially fast with $N$.

The following fact is also usefull:

Fact 4.8. Let $\left(Z_{N}\right)$ a symmetric random walk on a finitely generated group $\Gamma$, then for any fixed integer $N$ the function $\Gamma \rightarrow[0,1]: g \mapsto P\left(Z_{2 N}=g\right)$ is maximal for $g=i d_{\Gamma}$.

Proof of the Fact 4.8. Let $p_{k}(x, y)$ denote the probability to go from $x$ to $y$ in $k$ steps, let $\delta_{x}$ denote the function on $\Gamma$ taking values 1 on $x$ and 0 elsewhere and $M$ the symmetric random walk operator on the space $l^{2}(\Gamma)$. Then Cauchy inequality implies:

$$
\begin{aligned}
p_{2 N}(i d, x)^{2} & =\left\langle M^{2 N} \delta_{i d}, \delta_{x}\right\rangle^{2}=\left\langle M^{N} \delta_{i d}, M^{N} \delta_{x}\right\rangle^{2} \\
& \leq\left\|M^{N} \delta_{i d}\right\| .\left\|M^{N} \delta_{x}\right\|=p_{2 N}(i d, i d) \cdot p_{2 N}(x, x)=p_{2 N}(i d, i d)^{2} .
\end{aligned}
$$


Note that Theorem 4.7 and Fact 4.8 only apply to symmetric random walks.

Proof of Theorem 3.2. Given an arbitrary positive $\varepsilon$ the previous Fact 4.8 applied to the symmetric random walk $\left(Z_{N}\right)$ constructed above raises:

$$
P\left(\nu\left(Z_{2 N}\right) \leq \varepsilon 2 N\right)=\sum_{\nu(g) \leq \varepsilon 2 N} P\left(Z_{2 N}=g\right) \leq P\left(Z_{2 N}=i d_{G\left(S_{d_{0}}, H\right)}\right) \# B_{\nu}(\varepsilon 2 N),
$$

and the Propositions $4.1 \mathrm{~b})$ and 4.2 ensure:

$$
P\left(Z_{2 N}=i d\right) \geq P\left(\frac{\nu\left(Z_{2 N}\right)}{2 N} \leq \varepsilon\right) C^{-\varepsilon 2 N} \sim_{N \rightarrow \infty} C^{-\varepsilon 2 N} .
$$

Thus $P\left(Z_{2 N}=i d\right)$ does not decrease exponentially fast and Kesten's criterion proves Theorem 3.2 and thus the Main Theorem.

\section{Details of the proof of Theorem 3.2}

5.1. Fractal pseudo norms of exponential growth (proof of Proposition 4.1). To the symmetric generating set $S=\left(S_{d_{0}} \cup H\right) \backslash\{1\}$ of $G\left(S_{d_{0}}, H\right)$ is associated the word norm on $G\left(S_{d_{0}}, H\right)$ by:

$$
|g|=\min \left\{r \mid g=z_{1} \ldots z_{r}, z_{i} \in S\right\} .
$$

Denote $B_{S}(r)$ the ball of radius $r$ associated to this norm (that is the set of all $g$ such that $|g| \leq r)$, then $\# B_{S}(r) \leq(\# S)^{r}$.

Note that since $G\left(S_{d_{0}}, H\right)$ is a quotient of the free product $S_{d_{0}} * H$ a word $z_{1} \ldots z_{r}$ is a minimal representative of $g$ (that is $r=|g|$ ) only in the following cases: either $z_{2 j} \in S_{d_{0}} \backslash\{1\}$ and $z_{2 j+1} \in H \backslash\{1\}$, or conversely. This brings another definition:

$$
\|g\|_{0}=\min \left\{r \mid g=a_{1} h_{1} a_{2} h_{2} \ldots h_{r} a_{r+1}, a_{i} \in S_{d_{0}}, h_{j} \in H\right\} .
$$

The following is straightforward:

Properties 5.1. The function \|\|$_{0}$ is a norm when restricted to the stabilizer of the first level $S t_{1}\left(G\left(S_{d_{0}}, H\right)\right)$, namely it satisfies:

(1) $\|g h\|_{0} \leq\|g\|_{0}+\|h\|_{0}$ for all $g, h$ in $G\left(S_{d_{0}}, H\right)$,

(2) $\left\|g^{-1}\right\|_{0}=\|g\|_{0}$ for all $g$ in $G\left(S_{d_{0}}, H\right)$,

(3) $\|g\|_{0}=0$ if and only if $g \in S_{d_{0}}$,

This function $\|.\|_{0}$ is related to the usual word norm since for $g$ in $G\left(S_{d_{0}}, H\right)$ :

$$
2\|g\|_{0}-1 \leq|g| \leq 2\|g\|_{0}+1
$$

which implies that if $B_{\|.\|_{0}}(r)$ is the ball of radius $r$ associated to $\|.\|_{0}$ in $G\left(S_{d_{0}}, H\right)$, then:

$$
\# B_{\|\cdot\|_{0}}(r) \leq(\# S)^{2 r+1} .
$$

Following [BV], let us introduce a new function $\nu$ on $G\left(S_{d_{0}}, H\right)$ which is to be thought of as a fractal distance. For $g \in G\left(S_{d_{0}}, H\right)$ and a vertex $v$ on layer $k=l(v)$ of $T_{\bar{d}}$, denote by $g_{v}$ the action of $g$ on the descendant subtree $T_{v} \simeq T_{\sigma^{k} \bar{d}}$ of $T_{\bar{d}}$ and $g(v) \in S_{d_{k}}$ the action on the $d_{k}$ children of $v$. The automorphism $g_{v}$ of the 
rooted tree $T_{v}$ belongs to the group $G\left(S_{d_{k}}, H_{k}\right)$. The function defined by (5.1) for $G\left(S_{d_{k}}, H_{k}\right)$ will be denoted by $\|.\|_{k}$.

A subtree $T$ of $T_{\bar{d}}$ is said to be rooted if it contains the root $\emptyset$ of $T_{\bar{d}}$. It is said regular if for every vertex $v \in T$, either $T$ contains the $d_{l(n)}$ descendant of $v$, either it contains none of them.

Given a finite regular rooted subtree $T$ of $T_{\bar{d}}$ with set of leaves $\partial T$, define a function $\nu_{T}$ on $G\left(S_{d_{0}}, H\right)$ by:

$$
\nu_{T}(g)=\sum_{v \in \partial T}\left(1+\left\|g_{v}\right\|_{l(v)}\right) .
$$

and a function $\nu: G\left(S_{d_{0}}, H\right) \rightarrow \mathbb{N}$ as:

$$
\nu(g)=\min \left\{\nu_{T}(g) \mid T \text { is a finite regular rooted subtree of } T_{\bar{d}}\right\} .
$$

The construction (5.2) defines similarly a function $\nu^{k}: G\left(S_{d_{k}}, H_{k}\right) \rightarrow \mathbb{N}$ for the subgroup $G\left(S_{d_{k}}, H_{k}\right)<\operatorname{Aut}\left(T_{\sigma^{k} \bar{d}}\right) \simeq \operatorname{Aut}\left(T_{v}\right)$ for any vertex $v$ on the $k$ th layer. Note that $\nu=\nu^{0}$ and that the following proposition is still true replacing $\nu$ by $\nu^{k}$ and $\nu^{1}$ by $\nu^{k+1}$.

Proposition 5.2. The function $\nu$ satisfies:

(1) Let $g$ in $G\left(S_{d_{0}}, H\right)$ and $g=\left(g_{1}, \ldots, g_{d_{0}}\right) \sigma$ be its embedded image in the wreath product $G\left(S_{d_{0}}, H\right) \hookrightarrow G\left(S_{d_{1}}, H_{1}\right)$ \ $S_{d_{0}}$, then:

$$
\nu(g)=\min \left\{\nu^{1}\left(g_{1}\right)+\cdots+\nu^{1}\left(g_{d_{0}}\right), 1+\|g\|_{0}\right\} .
$$

(2) Let $g$ in $G\left(S_{d_{0}}, H\right)$, then $\nu(g)=\nu\left(g^{-1}\right)$.

(3) Let $g, g^{\prime}$ be in $G\left(S_{d_{0}}, H\right)$, then $\nu\left(g g^{\prime}\right) \leq\|g\|_{0}+\nu\left(g^{\prime}\right)$.

(4) Let $g, g^{\prime}$ be in $G\left(S_{d_{0}}, H\right)$, then $\nu\left(g g^{\prime}\right) \leq \nu(g)+\nu\left(g^{\prime}\right)$.

In particular, this function $\nu$ is a pseudo-norm on $G\left(S_{d_{0}}, H\right)$.

The use of induction in the proof of Proposition 5.2 requires the:

Property 5.3. Let $g$ in $G\left(S_{d_{k}}, H_{k}\right)$ have image $g=\left(g_{1}, \ldots, g_{d_{k}}\right) \sigma$ in the wreath product $G\left(S_{d_{k+1}}, H_{k+1}\right) \backslash S_{d_{k}}$ and assume $\|g\|_{k} \geq 2$ then $\left\|g_{t}\right\|_{k+1}<\|g\|_{k}$ for any coordinate $t$.

Proof of Property 5.3. An element $g$ admits a minimal representative of the form $g=h_{1}^{\sigma_{1}} \ldots h_{r}^{\sigma_{r}} \sigma_{r+1}$ with $\sigma_{i}$ in $S_{d_{k}}$ and $h_{i}$ in $H_{k}$ (remind $\left.x^{y}=y x y^{-1}\right)$. Moreover by construction $h=\left(h^{\prime}, a_{2}, \ldots, a_{d_{k}}\right)$ with $h^{\prime}$ in $H_{k+1}$ and $a_{i}$ in $S_{d_{k+1}}$ and the conjugate $h^{\sigma}$ is the same $d_{k}$-tuple where the coordinates are $\sigma$ permuted. This ensures $\left\|g_{1}\right\|_{k+1}+$ $\cdots+\left\|g_{d_{0}}\right\|_{k+1} \leq\|g\|_{k}$. It is sufficient to prove the property for $\|g\|_{k}=2$, that is $g=h_{1}^{\sigma_{1}} h_{2}^{\sigma_{2}}$. If $\sigma_{1}(1) \neq \sigma_{2}(1)$ the property is obvious. If $\sigma_{1}(1)=\sigma_{2}(1)$ then $\left\|g_{i}\right\|_{k+1}=0$ if $i \neq \sigma_{1}(1)$ and $\left\|g_{\sigma_{1}(1)}\right\|_{k+1}=\left\|h_{1}^{\prime} h_{2}^{\prime}\right\|_{k+1}=1$ because $h_{1}^{\prime} h_{2}^{\prime}$ is an element of $H_{k+1}$.

Proof of Proposition 5.2. Note that $1+\|g\|_{0}=\nu_{\{\emptyset\}}(g)$ and assume the minimum in definition (5.2) is obtained for a finite regular rooted tree $T \neq\{\emptyset\}$. Clearly 
$\partial T=\partial T(1) \cup \cdots \cup \partial T\left(d_{0}\right)$ where $T(v)$ denotes the intersection of $T$ with the subtree $T_{v}$ of $T_{\bar{d}}$ hung on vertex $v$, thus:

$$
\nu_{T}(g)=\sum_{v \in \partial T}\left(1+\left\|g_{v}\right\|_{l(v)}\right)=\sum_{t=1}^{d_{0}} \sum_{v \in \partial T(t)}\left(1+\left\|g_{v}\right\|_{l(v)}\right)=\sum_{t=1}^{d_{0}} \nu_{T(t)}\left(g_{t}\right),
$$

which is minimal if and only if $\nu_{T(t)}\left(g_{t}\right)=\nu^{1}\left(g_{t}\right)$ is minimal for all $t$. This implies part (1).

It follows that if $\|g\|_{0}=1$ then $\nu(g)=2=\nu\left(g^{-1}\right)$. Similarly if $\|g\|_{k}=1$ for $g \in G\left(S_{d_{k}}, H_{k}\right)$ then $\nu^{k}(g)=2=\nu^{k}\left(g^{-1}\right)$. Assume by induction on $r$ that $\nu^{k}(g)=\nu^{k}\left(g^{-1}\right)$ if $\|g\|_{k} \leq r$ and this jointly for every level $k$, then the inverse formula $g^{-1}=\sigma^{-1}\left(g_{1}^{-1}, \ldots, g_{d_{0}}^{-1}\right)$ and the induction hypothesis ensuring $\nu^{1}\left(\left(g^{-1}\right)_{1}\right)+$ $\cdots+\nu^{1}\left(\left(g^{-1}\right)_{d_{0}}\right)=\nu^{1}\left(g_{1}\right)+\cdots+\nu^{1}\left(g_{d_{0}}\right)$ (as $\left\|g_{t}\right\|_{k+1}<\|g\|_{k}$ by Property 5.3) together with part (1) show part (2).

To prove part (3), note first that $\nu(a g)=\nu(g)$ for all $a \in S_{d_{0}}$. Indeed, $a$ only permutes the subtrees of the first level and does not increase any of the $\left\|g_{v}\right\|_{l(v)}$. To conclude, it is sufficient to show that when $h$ is in $H$, we have $\nu_{T}(h g) \leq 1+\nu_{T}(g)$ for any finite regular subtree $T$. Proceed by induction on the size of $T$. Indeed, this is true for $T=\{\emptyset\}$ by Property 5.1 (1). More generally, denoting $g=\left(g_{1}, \ldots, g_{d_{0}}\right) \sigma_{0}$ and $h=\left(h_{1}, a_{2}, \ldots, a_{d_{0}}\right)$ with $g_{t}$ in $G\left(S_{d_{1}}, H_{1}\right), h_{1}$ in $H_{1}$ and $a_{t}$ in $S_{d_{1}}$, we get $h g=\left(h_{1} g_{1}, a_{2} g_{2}, \ldots, a_{d_{0}} g_{d_{0}}\right) \sigma_{0}$ and:

$$
\nu_{T}(h g)=\nu_{T(1)}\left(h_{1} g_{1}\right)+\sum_{t=2}^{d_{0}} \nu_{T(t)}\left(a_{t} g_{t}\right) \leq 1+\nu_{T(1)}\left(g_{1}\right)+\sum_{t=2}^{d_{0}} \nu_{T(t)}\left(g_{t}\right)=1+\nu_{T}(g)
$$

using the induction hypothesis on $T(1)$.

Part (4) is implied by part (3) in case $\nu(g)=1+\|g\|_{0}$ or $\nu\left(g^{\prime}\right)=1+\left\|g^{\prime}\right\|_{0}$. Otherwise:

$$
\nu\left(g g^{\prime}\right) \leq \sum_{t=1}^{d_{0}} \nu^{1}\left(\left(g g^{\prime}\right)_{t}\right)=\sum_{t=1}^{d_{0}} \nu^{1}\left(g_{t} g_{\sigma(t)}^{\prime}\right) \leq \sum_{t=1}^{d_{0}} \nu^{1}\left(g_{t}\right)+\nu^{1}\left(g_{\sigma(t)}\right)=\nu(g)+\nu\left(g^{\prime}\right),
$$

where the second inequality comes by joint induction on $\|g\|_{k}$ using Property 5.3.

Let $B_{\nu}(r)=\left\{g \in G\left(S_{d_{0}}, H\right) \mid \nu(g) \leq r\right\}$ denote the ball of radius $r$ associated to the function $\nu$. The next proposition is crucial for our purpose.

Proposition 5.4. Consider a spherically homogeneous rooted tree $T_{\bar{d}}$ of bounded valency $2 \leq d_{i} \leq D$, a finite subgroup $H$ of $\bar{H}$ and the function $\nu$ constructed above, then the balls $B_{\nu}(r) \subset G\left(S_{d_{0}}, H\right)$ grow at most exponentially fast. Namely, there exists a constant $C$ depending only on $D$ and the size of $H$ such that:

$$
\# B_{\nu}(r) \leq(C)^{r}, \quad \text { for all } r \text { sufficiently large. }
$$

In order to prove this proposition, recall classical estimates on the number of rooted subtrees of a rooted tree. The formula below can be found in $[\mathrm{PR}]$, the equivalent is derived from Stirling's formula. 
Proposition 5.5. The number of (not necessarily regular) rooted subtrees of a $D$ regular tree $T_{D}$ containing $r$ vertices is:

$$
s_{r}^{(D)}=\frac{1}{r} C_{D r}^{r-1} \sim_{r \rightarrow+\infty} \frac{1}{D-1} \sqrt{\frac{D}{2(D-1) \pi}} r^{-\frac{3}{2}}\left(\frac{D^{D}}{(D-1)^{(D-1)}}\right)^{r} .
$$

More precisely the following is sufficient:

Corollary 5.6. The number $t_{r}^{(D)}$ of regular rooted subtrees of $T_{\bar{d}}$ (with $\bar{d}$ bounded by D) containing at most $r$ leaves satisfies:

$$
t_{r}^{(D)} \leq\left(K_{D}\right)^{r}, \quad \text { for } K_{D}=\frac{D^{2 D}}{(D-1)^{2(D-1)}},
$$

provided $r$ is sufficiently large.

Proof. It is well known that a subtree with at most $r$ leaves contains at most $2 r-1$ vertices and the asymptotic equivalent of $s_{r}^{(D)}$ gives the corollary.

Proof of Proposition 5.4. If $\nu(g) \leq r$ then there exists a regular rooted subtree $T$ such that $\nu_{T}(g) \leq r$. In particular, such a subtree has less than $r$ leaves so that there are at most $\left(K_{D}\right)^{r}$ choices for $T$ (corollary 5.6). Given $T$, the element $g$ is described by all $g(v) \in S_{d_{l(v)}}$ where $v \in \stackrel{\circ}{T}$, which allow at most $(D !)^{\# \frac{\circ}{T}} \leq(D !)^{r}$ choices, and all $g_{v} \in G\left(S_{d_{l(v)}}, H_{l(v)}\right)$ with $v \in \partial T$, which satisfy:

$$
\sum_{v \in \partial T}\left\|g_{v}\right\|_{l(v)} \leq r
$$

The number of possibilities for this last choice is less than $(M+1)^{2 r}$ where $M=$ $\max \left\{\# B_{\|.\|_{k}}(1)\right\}$ (finite because the size of the generating set $S_{d_{k}} \cup H_{k}$ on layer $k$ depends only on $d_{k} \leq D$ and $\# H_{k} \leq \# H$ ) bounds the number of symbols which represent an automorphism of norm 1 on a given leaf. An extra symbol (a coma) is added to denote passing to the next leaf. All in all, taking $C=K_{D} D !(M+1)^{2}$ gives the desired result.

5.2. Similarity of random walks (proof of Lemma 4.6). First recall elementary probabilistic facts which will be usefull.

Fact 5.7. Let $\left(z_{i}\right)_{i \geq 1}$ be independent random variables equidistributed on a finite group $F$. Then the sequence $\left(X_{k}\right)_{k \geq 1}$ of products $X_{k}=z_{1} \ldots z_{k}$ is a family of independent random variables equidistributed on $F$.

Proof of Fact 5.7. Denote by $q_{F}$ the equidistribution measure on the finite group $F$. It is sufficient to prove by induction that:

$$
q_{F}^{\otimes \infty}\left(X_{i}=f_{i}, i \leq k\right)=\prod_{i=1}^{k} q_{F}^{\otimes \infty}\left(X_{i}=f_{i}\right)=\prod_{i=1}^{k} q_{F}\left(X_{i}=f_{i}\right),
$$


for arbitrary $f_{1}, \ldots, f_{k}$ in $F$, which comes from:

$$
\begin{aligned}
q_{F}^{\otimes \infty}\left(X_{i}=f_{i}, i \leq k\right) & =q_{F}^{\otimes \infty}\left(X_{k}=f_{k} \mid X_{i}=f_{i}, i \leq k-1\right) q_{F}^{\otimes \infty}\left(X_{i}=f_{i}, i \leq k-1\right) \\
& =q_{F}^{\otimes \infty}\left(z_{k}=f_{k-1}^{-1} f_{k}\right) \prod_{j=1}^{k-1} q_{F}^{\otimes \infty}\left(X_{j}=f_{j}\right) \\
& =q_{F}\left(z_{k}=f_{k-1}^{-1} f_{k}\right) \prod_{j=1}^{k-1} q_{F}\left(X_{j}=f_{j}\right) .
\end{aligned}
$$

Fact 5.8. Let $z$ be a random variable equidistributed on a finite group $F$ acting transitively on a finite set $A$, then $q_{F}\left(z(t)=t^{\prime}\right)=\frac{1}{\# A}$ for all $t, t^{\prime}$ in $A$.

Proof of Fact 5.8. The quotient $F / S t a b_{F}(t)$ is of size $\# A$. If $z_{0}(t)=t^{\prime}$ (transitivity) then $z_{0} S t a b_{F}(t)=\left\{z \mid z(t)=t^{\prime}\right\}$ has the same size as $S t a b_{F}(t)$ by injectivity of left translation in $F$.

Fact 5.9. Let $\left(u_{i}\right)_{i \in \mathbb{N}}$ be independent random Bernoulli variables on $\{0,1\}$ (say $p\left(u_{i}=0\right)=p$ and $p\left(u_{i}=1\right)=1-p$ for some $p$ in $] 0,1[)$. Let $f\left(w_{N}\right)$ be the number of alternations in the subsequence $w_{N}=u_{1} \ldots u_{N}$, that is the number of indexes $i$ such that $u_{i} \neq u_{i+1}$. Equivalently, $1+f\left(w_{N}\right)$ is the number of maximal packs of constant successive terms. Then:

$$
f\left(w_{N}\right) \sim_{N \rightarrow+\infty} 2 p(1-p) N, \quad P=p^{\otimes \infty} \text { a.s. }
$$

Proof of Fact 5.9. Apply the law of large numbers to $f\left(w_{N}\right)=\sum_{i=1}^{N-1} 1_{\left\{u_{i} \neq u_{i+1}\right\}}$ knowing that $E\left(1_{\left\{u_{i} \neq u_{i+1}\right\}}\right)=2 p(1-p)$ and that the terms are independent.

Proof of Lemma 4.6. Consider the random walk $Y_{n}$ at step $n$ as:

$$
Y_{n}=t_{0} t_{1} \ldots t_{n}=a_{1} h_{1} a_{2} h_{2} \ldots a_{s} h_{s} a_{s+1}
$$

with $s=\left[\frac{n}{2}\right]\left(a_{s+1}\right.$ empty if $n$ even), where the terms $a_{i}$ (resp. $\left.h_{i}\right)$ are random variables equidistributed in $S_{d_{0}}$ (resp. in $H$ ), all being independent. This can be rewritten $Y_{n}=h_{1}^{\sigma_{1}} \ldots h_{s}^{\sigma_{s}} \sigma_{s+1}$ (remind the conjugate notation $h^{\sigma}=\sigma h \sigma^{-1}$ ) where the $\sigma_{i}=a_{1} a_{2} \ldots a_{i}$ are independent random variables equidistributed in $S_{d_{0}}$ by Fact 5.7.

Using coordinates in the wreath product an element $h$ of $H$ has the form $h=$ $\left(h^{1}, a^{2}, \ldots, a^{d_{0}}\right)$ with $h^{1}$ in $H_{1}$ and $a^{i}$ in $S_{d_{1}}$ and each of them is equidistributed for $h$ equidistributed in $H$ by saturation (note that the coordinates are not independent). Conjugating by a rooted automorphism $\sigma$ raises $h^{\sigma}=\left(a^{\sigma(1)}, \ldots, a^{\sigma\left(d_{0}\right)}\right)$ with $h^{1}$ in position $\sigma(1)$.

Consider now the random walk $Y_{n}=\left(Y_{n}^{1}, \ldots, Y_{n}^{d_{0}}\right) \sigma_{n}$ at time $n$ and focus on coordinate $t$, which is a product $Y_{n}^{t}=u_{1} \ldots u_{s}$ of $s$ independent terms such that $u_{i}$ belongs to and is equidistributed in $S_{d_{1}}$ (resp. $H_{1}$ ) if $\sigma_{i}(t)$ belongs to $\left\{2, \ldots, d_{0}\right\}$ (resp. $\sigma_{i}(t)=1$ ). Since the $\sigma_{i}$ are equidistributed in $S_{d_{0}}$ the probability that $u_{i}$ is 
in $S_{d_{1}}$ (resp. $\left.H_{1}\right)$ for a given $i$ is $\frac{d_{0}-1}{d_{0}}$ (resp. $\frac{1}{d_{0}}$ ) by Fact 5.8 . This is summarized in:

$$
Q\left(u_{i}=g\right)= \begin{cases}\frac{d_{0}-1}{d_{0}} \frac{1}{\# S_{d_{1}}} & \text { if } g \in S_{d_{1}}, \\ \frac{1}{d_{0}} \frac{1}{\# H_{1}} & \text { if } g \in H_{1},\end{cases}
$$

and the terms $u_{i}$ are independent. Define $m_{t}(n)$ to be the number of maximal packs of successive $u_{i}$ belonging either to $S_{d_{1}}$, or to $H_{1}$ in the sequence $Y_{n}^{t}=u_{1} \ldots u_{s}$. Fact 5.9 ensures that:

$$
m_{t}(n) \sim_{n \rightarrow+\infty} 2 \frac{1}{d_{0}}\left(1-\frac{1}{d_{0}}\right) s \sim_{n \rightarrow+\infty}\left(\frac{d_{0}-1}{d_{0}}\right) \frac{n}{d_{0}} .
$$

Given an integer $n$, assume we know the distribution $\mathcal{D}$ of which terms $u_{i}$ are in $S_{d_{1}}$ and $H_{1}$, then the $k$ th pack of terms $v_{k}=u_{i_{k}} u_{i_{k}+1} \ldots u_{j_{k}}$ of constant belonging is a product of equidistributed independent elements in the finite group $S_{d_{1}}$ or $H_{1}$ hence is equidistributed. In this situation $Y_{n}^{t}=v_{0} v_{1} \ldots v_{m_{t}(n)}$ where two cases are possible: either $u_{1}$ belongs to $S_{d_{1}}\left(\right.$ set $\left.\varepsilon_{t}(n)=0\right)$, the terms $v_{2 k+1}$ are equidistributed in $H_{1}$ and $v_{2 k}$ are equidistributed in $S_{d_{1}}$, which is of the form $Y_{m_{t}(n)}^{\prime}$; or $u_{1}$ belongs to $H_{1}$ (set $\varepsilon_{t}(n)=1$ ), then re index the $v_{i}$ as $Y_{n}^{t}=i d_{S_{d_{1}}} v_{1} \ldots v_{m_{t}(n)+1}$ which is of the form $Y_{m_{t}(n)+1}^{\prime}$ except for $v_{0}$ which follows the Dirac law on $i d_{S_{d_{1}}}$; this has no influence on the $\nu$-distribution of the sequences (Remark 4.4). In both cases:

$$
\left(Y_{n}^{t} \mid \mathcal{D}\right) \sim_{\nu \text { law }} Y_{m_{t}(n)+\varepsilon_{t}(n)}^{\prime},
$$

where the condition depends only on the number of alternations $m_{t}(n)$ and the starting condition $\varepsilon_{t}(n)$ of the distribution $\mathcal{D}$.

5.3. Zero drift of $\left(Y_{n}\right)$ (proof of Proposition 4.3). First note that the Kolmogorov 01-law implies almost sure constance of $\lim \sup \frac{\nu\left(Y_{n}\right)}{n}$.

Lemma 5.10. For every integer $k$ denote $\left(Y_{n}^{(k)}\right)_{n}$ the random walk on $G\left(S_{d_{k}}, H_{k}\right)$ which is taking independent equidistributed increments alternatively in $S_{d_{k}}$ and $H_{k}$, in particular $\left(Y_{n}\right)=\left(Y_{n}^{(0)}\right)$ and $\left(Y_{n}^{\prime}\right)=\left(Y_{n}^{(1)}\right)$. Then there exists $l_{k}$ in $\left[0, \frac{1}{2}\right]$ such that:

$$
\limsup _{n \rightarrow+\infty} \frac{\nu^{k}\left(Y_{n}^{(k)}\right)}{n}=l_{k}, \quad Q_{k}=\left(q_{S_{d_{k}}} \otimes q_{H_{k}}\right)^{\otimes \infty} \text { a.s.. }
$$

Proof. Proposition $5.2(1)$ implies $\nu^{k}\left(Y_{n}^{(k)}\right) \leq 1+\left\|Y_{n}^{(k)}\right\|_{k} \leq \frac{n+1}{2}$ so that the limsup is $\leq \frac{1}{2}$. Given $l$ in $\left[0, \frac{1}{2}\right]$ the event $E_{l}=\left\{\limsup \frac{\nu^{k}\left(Y_{n}^{(k)}\right)}{n} \leq l\right\}$ is a tail event, that is an event which is independent of any finite subsequence $\left(Y_{n}^{(k)}\right)_{n \leq N}$, hence has probability 0 or 1 by the 01-Kolmogorov law. The function $l \mapsto Q_{k}\left(E_{l}\right)$ is increasing, right continuous and takes values in $\{0,1\}$, so that there exists $l_{k}$ such that $Q_{k}\left(E_{l}\right)=0$ for $l<l_{k}$ and $Q_{k}\left(E_{l}\right)=1$ for $l \geq l_{k}$. Then:

$$
Q_{k}\left(\left\{\limsup \frac{\nu^{k}\left(Y_{n}^{(k)}\right)}{n}=l_{k}\right\}\right)=Q_{k}\left(E_{l_{k}} \backslash \cup_{n \geq 1} E_{l_{k}-\frac{1}{n}}\right)=1 .
$$


Proof of Proposition 4.3. To show $l_{0}=0$, prove $l_{k} \leq \frac{(D-1)}{D} l_{k+1}$ where $D$ is the bound on the valencies of the spherically homogeneous rooted tree $T_{\bar{d}}$. This is sufficient as $l_{k} \leq \frac{1}{2}$ for every $k$. To ease notations, compute for $k=0$. Proposition 4.1 (a) ensures:

$$
\limsup _{n \rightarrow+\infty} \frac{\nu\left(Y_{n}\right)}{n} \leq \limsup _{n \rightarrow+\infty} \sum_{t=1}^{d_{0}} \frac{\nu^{1}\left(Y_{n}^{t}\right)}{n} \leq \sum_{t=1}^{d_{0}} \limsup _{n \rightarrow+\infty} \frac{\nu^{1}\left(Y_{n}^{t}\right)}{n} .
$$

To compute the right side introduce the condition $\left(m_{t}(n)\right)$ :

$$
\limsup _{n \rightarrow+\infty} \frac{\nu^{1}\left(Y_{n}^{t}\right)}{n}=\limsup _{n \rightarrow+\infty} \frac{\nu^{1}\left(Y_{n}^{t}\right)}{m_{t}(n)} \frac{m_{t}(n)}{n} \leq \limsup _{n \rightarrow+\infty} \frac{\nu^{1}\left(Y_{n}^{t}\right)}{m_{t}(n)} \limsup _{n \rightarrow+\infty} \frac{m_{t}(n)}{n},
$$

where Lemma 4.6 gives $\lim \sup \frac{m_{t}(n)}{n}=\left(\frac{d_{0}-1}{d_{0}}\right) \frac{1}{d_{0}}, Q$ a.s. and

$$
\limsup \frac{\nu^{1}\left(Y_{n}^{t}\right)}{m_{t}(n)}=\limsup \frac{\nu^{1}\left(Y_{m_{t}(n)+\varepsilon_{n}^{t}}^{\prime}\right)}{m_{t}(n)}=l_{1}, \quad Q \text { a.s. }
$$

because $m_{t}(n) \rightarrow+\infty Q$ a.s.. The last estimates gathered together on a $Q$ probability one event show that:

$$
l_{0} \leq \sum_{t=1}^{d_{0}} l_{1}\left(\frac{d_{0}-1}{d_{0}}\right) \frac{1}{d_{0}}=\left(\frac{d_{0}-1}{d_{0}}\right) l_{1} \leq\left(\frac{D-1}{D}\right) l_{1} .
$$

\subsection{Zero drift of $\left(Z_{N}\right)$ (proof of Proposition 4.2). Recall the:}

Fact 5.11. Let $\left(a_{i}\right)_{i \in \mathbb{N}}$ be a random sequence in $\{0,1\}^{\mathbb{N}}$ endowed with a probability measure $\mu$. Assume that there exists an infinite subset $I$ of $\mathbb{N}$ such that $\mu\left(a_{i}=1\right) \geq$ $\delta>0$ for all $i \in I$, then $\mu\left(a_{i}=1\right.$ for infinitely many $\left.i\right) \geq \delta$.

Proof of Fact 5.11. Let $E=\left\{\left(a_{i}\right) \mid a_{i}=1\right.$ infinitely often $\}$ and assume by contradiction $\mu(E)=\delta^{\prime}<\delta$, this implies $\mu\left(E^{c} \cap\left\{a_{i}=1\right\}\right) \geq \delta-\delta^{\prime}$ for all $i$ in $I$. However the complement of $E$ is the infinite increasing union:

$$
E^{c}=\cup_{n \in \mathbb{N}}\left\{\left(a_{i}\right) \mid a_{i}=0 \text { for } i \geq n\right\}=\cup_{n \in \mathbb{N}} F_{n},
$$

so that $\mu\left(F_{N}\right) \geq 1-\frac{\delta+\delta^{\prime}}{2}$ for some $N$. But the case $i \geq N$ raises the contradiction:

$$
\mu\left(E^{c} \cap\left\{a_{i}=1\right\}\right)=\mu\left(\left(E^{c} \backslash F_{N}\right) \cap\left\{a_{i}=1\right\}\right) \leq \mu\left(E^{c} \backslash F_{N}\right) \leq \frac{\delta+\delta^{\prime}}{2}-\delta^{\prime}=\frac{\delta-\delta^{\prime}}{2} .
$$

Proposition 4.3 will be used in the (a priori) weaker form:

Corollary 5.12. For every positive $\varepsilon$ and $\alpha$, there exists $N_{0}$ such that for $n \geq N_{0}$ :

$$
Q\left(\frac{\nu\left(Y_{n}\right)}{n} \leq \varepsilon\right) \geq 1-\alpha
$$

Proof. Assume the statement does not hold, then there exists $\varepsilon_{0}, \alpha_{0}$ and infinitely many integers $n_{k}$ with $Q\left(\frac{\nu\left(Y_{n_{k}}\right)}{n_{k}} \geq \varepsilon_{0}\right) \geq \alpha_{0}$ and then $Q\left(\lim \sup \frac{\nu\left(Y_{n}\right)}{n} \geq \varepsilon_{0}\right) \geq \alpha_{0}$ by Fact 5.11, contradicting Proposition 4.3. 
The random walks $\left(Z_{N}\right)$ and $\left(Y_{n}\right)$ are closely related by:

Fact 5.13. Let $N$ be a fixed integer. To each walk $Z_{N}=s_{1} \ldots s_{N}$ is associated the number of alternations a $(N)$ from $s_{i}$ in $S_{d_{0}}$ to $s_{i+1}$ in $H$ or vice versa. Then the conditional law of $Z_{N}$ satisfies:

$$
\left(Z_{N} \mid a(N)\right) \sim_{\nu-l a w} Y_{a(N)} .
$$

Proof. Conditioning by the distribution $\mathcal{D}$ of which terms $s_{i}$ are in $S_{d_{0}}$ and in $H$, the walk is rewritten: $Z_{N}=s_{1} \ldots s_{i_{0}} s_{i_{0}+1} \ldots s_{i_{1}} \ldots s_{i_{a(N)}}=t_{0} t_{1} \ldots t_{a(N)}$ where $t_{2 j}=$ $s_{i_{2 j-1}} \ldots s_{i_{2 j}}$ are equidistributed in $S_{d_{0}}$ (except maybe $t_{0}$ which could be empty) and $t_{2 j+1}=s_{i_{2 j}} \ldots s_{i_{2 j+1}}$ are equidistributed in $H$, all factors being independent, which is the definition of the random walk $Y_{a(N)}$. The condition matters only on $a(N)$ and not $\mathcal{D}$.

This Fact 5.13 allows us to show a weak form:

Lemma 5.14. For every positive $\varepsilon$ and $\alpha$, there exists $M$ such that for $N \geq M$ :

$$
P\left(\frac{\nu\left(Z_{N}\right)}{N} \leq \varepsilon\right) \geq 1-\alpha
$$

Proof. Fact 5.9 ensures that the conditioning term $a(N)$ satisfies $\lim _{N \rightarrow \infty} \frac{a(N)}{N}=\frac{1}{2}$, $P$ almost surely. In particular for every positive $\alpha$ there exists an integer $N_{1}$ such that $P\left(a(N) \geq \frac{N}{3}\right) \geq 1-\alpha$ for all $N \geq N_{1}$.

Now compute under the condition $a(N)$ :

$$
P\left(\frac{\nu\left(Z_{N}\right)}{N} \leq \varepsilon\right)=\sum_{a(N)} P\left(\frac{\nu\left(Z_{N}\right)}{N} \leq \varepsilon \mid a(N)\right) P(a(N)),
$$

but if $N \geq N_{1}$ then $P\left(a(N) \leq \frac{N}{3}\right) \leq \alpha$. Moreover for $N \geq 3 N_{0}$ (defined by Corollary 5.12) the condition $a(N) \geq \frac{N}{3} \geq N_{0}$ ensures via Fact 5.13:

$$
P\left(\frac{\nu\left(Z_{N}\right)}{N} \leq \varepsilon \mid a(N)\right)=Q\left(\frac{\nu\left(Y_{a(N)}\right)}{a(N)} \frac{a(N)}{N} \leq \varepsilon\right) \geq 1-\alpha,
$$

because $\frac{a(N)}{N} \leq 1$. All in all, when $N \geq \max \left\{N_{1}, 3 N_{0}\right\}$ :

$$
P\left(\frac{\nu\left(Z_{N}\right)}{N} \leq \varepsilon\right) \geq \sum_{a(N) \geq \frac{N}{3}}(1-\alpha) P(a(N)) \geq(1-\alpha)^{2},
$$

which proves Lemma 5.14.

The previous Lemma ensures that $P$ almost surely: $\lim \inf \frac{\nu\left(Z_{N}\right)}{N}=0$ (Fact 5.11). To get Proposition 4.2 use:

Theorem 5.15 (Kingman subadditive Theorem ([Kal] 9.14)). Let $\left(X_{m, n}\right)$ be random variables such that:

(1) $X_{0, n} \leq X_{0, m}+X_{m, n}$ for all $0<m<n$,

(2) $\left(X_{m+1, n+1}\right)$ has the same law as $\left(X_{m, n}\right)$,

(3) $E\left(X_{0,1}^{+}\right)<+\infty$, 
then the random sequence $\left(\frac{X_{0, n}}{n}\right)$ converges almost surely.

Applying this to $X_{n, m}=\nu\left(Z_{m}^{-1} Z_{n}\right)$ shows that the inferior limit is in fact a limit, proving Proposition 4.2. The interested reader will remark that Lemma 5.14 is sufficient for our purpose and thus the Main Theorem does not rely on Kingman's Theorem.

\section{Groups of intermediate Growth}

6.1. Generating pairs for alternate groups. In his paper [Wil2] (Proposition 2.1), Wilson constructs interesting generating pairs of alternate groups $\mathcal{A}_{d}$ :

Proposition 6.1 (Wilson [Wil2]). Let $d \geq 29$, then the alternate group of permutation $\mathcal{A}_{d}$ of the finite set $\{1, \ldots, d\}$ contains an eligible (see [Wil2] for the full definition) pair of elements $x_{d}, y_{d}$. In particular:

1) the pair is generating: $\left\langle x_{d}, y_{d}\right\rangle=\mathcal{A}_{d}$, the elements have order 2 and 3: $x_{d}^{2}=$ $y_{d}^{3}=1$, and a fixed point property that there exists $\alpha$ and $\beta$ in $\{1, \ldots, d\}$ such that: $x_{d}(\alpha)=y_{d} x_{d} y_{d}^{-1}(\alpha)=\alpha$ and $y_{d}(\beta)=\beta$ (up to re index we assume $\alpha=1$ and $\beta=2$ ).

2) let $\hat{x}=(u, 1, \ldots, 1) x_{d_{0}}$ and $\hat{y}=(1, v, 1, \ldots, 1) y_{d_{0}}$ belong to Aut $\left(T_{\bar{d}}\right)$ with $d_{0} \geq 29$ and $u, v$ in $A u t\left(T_{\sigma \bar{d}}\right)$ with $u^{2}=v^{3}=1$, then the group generated by $\hat{x}$ and $\hat{y}$ contains the whole group of alternate rooted automorphisms $\mathcal{A}_{d_{0}}$. More precisely:

$$
\langle\hat{x}, \hat{y}\rangle \simeq\langle u, v\rangle ح \mathcal{A}_{d_{0}}
$$

Given a (not necessarily bounded) sequence $\bar{d}$ of integers $\geq 29$, the above Proposition 6.1 allows to define recursively the following pair of automorphisms of the spherically homogeneous rooted tree $T_{\bar{d}}$ (remind the assumption on fixed points $\alpha=1$ and $\beta=2$ ):

$$
\begin{aligned}
& x_{\bar{d}}=\left(x_{\sigma \bar{d}}, 1, \ldots, 1\right) x_{d_{0}}, \\
& y_{\bar{d}}=\left(1, y_{\sigma \bar{d}}, 1, \ldots, 1\right) y_{d_{0}} .
\end{aligned}
$$

This definition is best understood by looking at the portraits on Figure 3. The automorphism subgroup generated is denoted $H_{\bar{d}}=\left\langle x_{\bar{d}}, y_{\bar{d}}\right\rangle$. Note that in the case $\bar{d}=\sigma \bar{d}$ is a constant sequence the group $H_{\bar{d}}$ is generated by a two (non trivial) state automaton.

Property 6.2. The alternate automorphism $x_{\bar{d}}$ has order 2 , and $y_{\bar{d}}$ has order 3.

Proof. Show by joint (on $x_{\sigma^{i} \bar{d}}$ for $i$ in $\mathbb{N}$ ) induction on $k$ that $x_{\sigma^{i} \bar{d}}^{2}$ acts trivially on the $k$ first levels of $T_{\sigma^{i} \bar{d}}$. This implies it acts trivially on the whole tree hence is trivial automorphism. Proposition 6.1 1) ensures:

$$
x_{\sigma^{i} \bar{d}}^{2}=\left(x_{\sigma^{i} \bar{d}}^{2}, 1, \ldots, 1\right) x_{d_{i}}^{2}=\left(x_{\sigma^{i} \bar{d}}^{2}, 1, \ldots, 1\right),
$$

which initiates the induction. Moreover $x_{\sigma^{i} \bar{d}}^{2}$ acts trivially on the subtrees $T_{2}, \ldots, T_{d_{i}}$ of $T_{\sigma^{i} \bar{d}}$ and as $x_{\sigma^{i+1} \bar{d}}^{2}$ on $T_{1}$ which acts trivially on the $k$ first level of $T_{1}$ by induction hypothesis. This proves $x_{\sigma^{i} \bar{d}}^{2}$ acts trivially on the $k+1$ first levels of $T_{\sigma^{i} \bar{d}}$. 

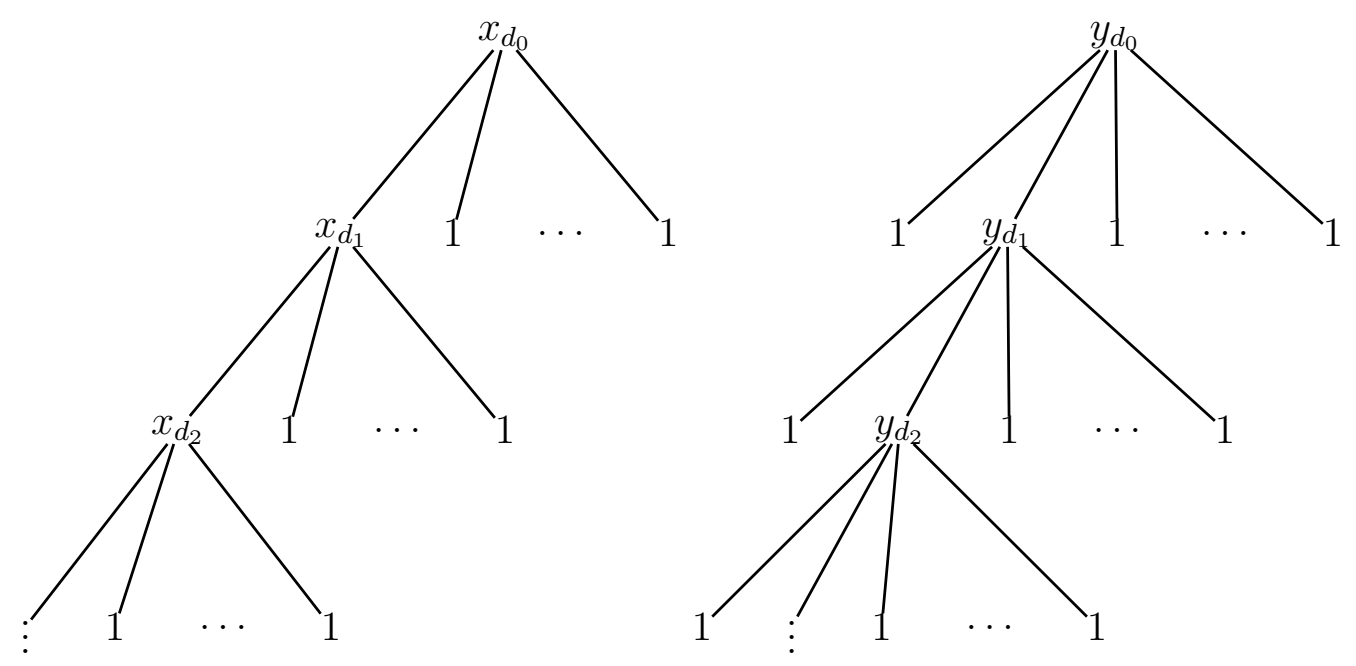

Figure 3. Portraits of the elements $x_{\bar{d}}$ and $y_{\bar{d}}$.

\subsection{Density properties.}

Proposition 6.3. The subgroup $H_{\bar{d}}=\left\langle x_{\bar{d}}, y_{\bar{d}}\right\rangle\left\langle A u t^{e}\left(T_{\bar{d}}\right)\right.$ is dense in $A u t^{e}\left(T_{\bar{d}}\right)$ endowed with the profinite topology from (2.4).

Proof. It is sufficient to show that the subgroup $\left.\mathcal{A}_{d_{k}} \prec \cdots\right\urcorner \mathcal{A}_{d_{0}}<A u t^{e}\left(T_{\bar{d}}\right)$ of alternate automorphisms of portrait supported on the $k$ firsts levels is included in $H_{\bar{d}}$ for arbitrary $k$. Proceed by joint (on $H_{\sigma^{i} \bar{d}}$ for $i \in \mathbb{N}$ ) induction on $k$ to show:

$$
H_{\sigma^{i} \bar{d}} \simeq H_{\sigma^{i+k} \bar{d}}>\mathcal{A}_{d_{i-1}} \prec \cdots 2 \mathcal{A}_{d_{i}}
$$

which will be sufficient taking $i=0$ and the trivial subgroup of $H_{\sigma^{k}} \bar{d}$. The case $k=0$ follows from Proposition 6.12 ):

$$
H_{\sigma^{i} \bar{d}}=\left\langle x_{\sigma^{i} \bar{d}}, y_{\sigma^{i} \bar{d}}\right\rangle \simeq\left\langle x_{\sigma^{i+1} \bar{d}}, y_{\sigma^{i+1} \bar{d}}\right\rangle\left\langle\mathcal{A}_{d_{i}}=H_{\sigma^{i+1} \bar{d}} \prec \mathcal{A}_{d_{i}} .\right.
$$

Assuming isomorphism (6.2) then isomorphism (6.3) for $i+k$ proves step $k+1$ :

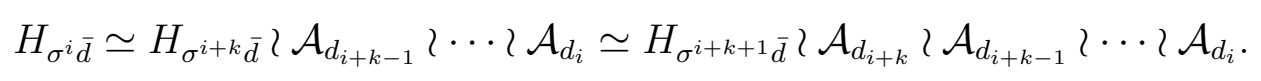

This density property is in contrast with the case of the full (non alternate) automorphism group of a rooted tree:

Proposition 6.4. The group Aut $\left(T_{\bar{d}}\right)$ endowed with the profinite topology from (2.3) admits no finitely generated dense subgroup.

Proof. Denote sgn $: S_{d} \rightarrow \mathbb{Z} / 2 \mathbb{Z}$ the signature morphism of permutations. Given an element $g$ in $\operatorname{Aut}\left(T_{\bar{d}}\right)$, recall that $g(v)$ is the permutation in $S_{d_{l(v)}}$ associated to vertex $v$ in the portrait of $g$.

(Recall $g=\left(g_{1 \ldots 1}, \ldots, g_{v}, \ldots, g_{d_{0} \ldots d_{l(v)-1}}\right) \tau_{l(v)-1}$ with $g_{v}$ in $\operatorname{Aut}\left(T_{v}\right) \simeq \operatorname{Aut}\left(T_{\sigma^{l(v)} \bar{d}}\right)$ and $\tau_{l(v)-1} \in \operatorname{Aut}\left(T_{d_{0} \ldots d_{l(v)-1}}\right)$, then $g_{v}$ has image $g_{v}=\left(g_{v 1}, \ldots, g_{v d_{l(v)}}\right) g(v)$ via the isomorphism Aut $\left.\left(T_{\sigma^{l(v)} \bar{d}}\right) \simeq \operatorname{Aut}\left(T_{\sigma^{l(v)+1} \bar{d}}\right)<S_{d_{l(v)}}.\right)$ 
Similarly to Lemma 1. in [Ale2], define for each integer $k$ the following morphism (of products of signatures of permutations on level $k$ in the portraits):

$$
\begin{aligned}
R_{k}: \operatorname{Aut}\left(T_{\bar{d}}\right) & \rightarrow \mathbb{Z} / 2 \mathbb{Z} \\
g & \mapsto R_{k}(g)=\prod_{v \in \operatorname{Level}(k)} \operatorname{sgn}(g(v)) .
\end{aligned}
$$

The computations via the isomorphism (2.2) show this is a group morphism. The product morphism $\varphi: \operatorname{Aut}\left(T_{\bar{d}}\right) \rightarrow(\mathbb{Z} / 2 \mathbb{Z})^{\infty}$ defined as $\varphi(g)=\left(R_{0}(g), R_{1}(g), \ldots\right)$ is then a surjective group morphism continuous for the profinite topologies. Assume now there exists a finitely generated dense subgroup $G$ of $\operatorname{Aut}\left(T_{\bar{d}}\right)$, then $\varphi(G)$ is a finitely generated dense subgroup of $(\mathbb{Z} / 2 \mathbb{Z})^{\infty}$. This is impossible since any finitely generated subgroup of $(\mathbb{Z} / 2 \mathbb{Z})^{\infty}$ is finite thanks to Fact 3.4 .

Density in $A u t^{e}\left(T_{\bar{d}}\right)$ of a finitely generated subgroup implies superpolynomial growth:

Proposition 6.5. Let $\bar{d}=\left(d_{i}\right)_{i \in \mathbb{N}}$ a sequence of integers $d_{i} \geq 3$, then any dense finitely generated subgroup of $A u t^{e}\left(T_{\bar{d}}\right)$ has superpolynomial growth.

Proof. Let $G$ be such a group and $k$ an arbitrary integer, then the level $k$ stabilizer $S t_{k}(G) \simeq G_{1 \ldots 1} \times \cdots \times G_{d_{0} \ldots d_{k-1}}$ is a direct product of $d_{0} \ldots d_{k-1}$ subgroups of $A u t^{e}\left(T_{\sigma^{k}} \bar{d}\right)$ each of which inherits the property to be dense and finitely generated. In particular each of the groups $G_{v}$ is infinite $\left(d_{i} \geq 3\right)$ and thus has at least linear growth, so that the subgroup $S t_{k}(G)$ of finite index and thus $G$ have growth function at least $b(r) \succsim r^{d_{0} \ldots d_{k-1}}$, hence superpolynomial.

\subsection{Intermediate growth.}

Proposition 6.6. The group $H_{\bar{d}}<A u t\left(T_{\bar{d}}\right)$ has intermediate growth.

Proof of Proposition 6.6. Superpolynomial growth follows from Propositions 6.3 and 6.5, so there remains to prove subexponential growth. Proceed as in [Gri1]. Denote $B_{k}(r)$ the ball of radius $r$ in $H_{\sigma^{k}} \bar{d}$ for the word metric $|\cdot|_{k}$ associated with the generating set $\left\langle x_{\sigma^{k}}, y_{\sigma^{k}}\right\rangle$, denote $b_{k}(r)$ its cardinal and $c_{k}=\lim \sqrt[r]{b_{k}(r)}=$ $h_{\left\{x_{\sigma_{\bar{d}}}, y_{\sigma_{\bar{d}}}\right\}}\left(H_{\sigma^{k} \bar{d}}\right)$ its exponential growth rate. The fixed point condition on eligible pairs ensures:

$$
x_{\bar{d}} y_{\bar{d}} x_{\bar{d}} y_{\bar{d}}^{-1} x_{\bar{d}}=\left(x_{\sigma \bar{d}}, 1, \ldots, y_{\sigma \bar{d}}, y_{\sigma \bar{d}}^{-1}, \ldots, 1\right) x_{d_{0}} y_{d_{0}} x_{d_{0}} y_{d_{0}}^{-1} x_{d_{0}},
$$

with $y_{\sigma \bar{d}}$ in positions $x_{d_{0}}(2)$ and $x_{d_{0}} y_{d_{0}} x_{d_{0}}(2)$ and the second and third $x_{\sigma \bar{d}}$ cancel out. As the generators are of order 2 and 3 every element $g=\left(g_{1}, \ldots, g_{d_{0}}\right) \sigma$ in $B_{0}(r)$ admits a minimal representative word of the form $g=x_{\bar{d}} y_{\bar{d}}^{\varepsilon_{1}} x_{\bar{d}} y_{\bar{d}}^{\varepsilon_{2}} \ldots x_{\bar{d}} y_{\bar{d}}^{\varepsilon_{n}} x_{\bar{d}}$, with $\varepsilon_{i}$ in $\{-1,1\}$. Given $g$ (more precisely given a fixed minimal representative word), denote $a(g)$ the number of alternations in the sequence $\left(\varepsilon_{i}\right)$, equality (6.4) implies:

$$
\left|g_{1}\right|_{1}+\cdots+\left|g_{d_{0}}\right|_{1} \leq|g|_{0}-a(g) .
$$

Given any parameter $t \geq 2$, split the ball $B_{0}(r)$ into:

$$
\begin{aligned}
& B_{0}^{+}(r)=\left\{g \in B_{0}(r) \mid a(g) \geq \frac{r}{t}\right\}, \\
& B_{0}^{-}(r)=\left\{g \in B_{0}(r) \mid a(g) \leq \frac{r}{t}\right\} .
\end{aligned}
$$


The size of the first part of the ball is bounded by:

$$
b_{0}^{+}(r) \leq \# \mathcal{A}_{d_{0}} \sum_{r_{1}+\cdots+r_{d_{0}} \leq\left(1-\frac{1}{t}\right) r} b_{1}\left(r_{1}\right) \ldots b_{1}\left(r_{d_{0}}\right) .
$$

Indeed, each element $g=\left(g_{1}, \ldots, g_{d_{0}}\right) \sigma$ of $B_{0}(r)$ is injectively described by the permutation $\sigma$ in $\mathcal{A}_{d_{0}}$ and the coordinates $g_{1}, \ldots, g_{d_{0}}$ the sum of the $|\cdot|_{1}$ length is bounded by $r-a(g) \leq\left(1-\frac{1}{t}\right) r$ thanks to computation (6.5). The size of the second part of the ball is bounded by (recall notation $C_{n}^{k}$ for the number of subsets of size $k$ in $\{1, \ldots, n\})$ :

$$
b_{0}^{-}(r) \leq 4 \sum_{s \leq \frac{r}{t}} C_{r}^{s} \leq 4 \frac{r}{t} C_{r}^{\frac{r}{t}}
$$

Indeed the term 4 corresponds to choosing the start of the representative word $(y$, $y^{-1}, x y$ or $\left.x y^{-1}\right), s$ represents the number of alternation $a(g)$ and $C_{r}^{s}$ the number of choice for the positions of such alternations.

The size is estimated by $b_{0}(r) \leq b_{0}^{+}(r)+b_{0}^{-}(r) \leq \max \left\{2 b_{0}^{+}(r), 2 b_{0}^{-}(r)\right\}$, and taking limits of $r$-roots raises $c_{0} \leq \max \left\{c_{1}^{1-\frac{1}{t}}, t^{\frac{1}{t}}\left(1-\frac{1}{t}\right)^{\left(\frac{1}{t}-1\right)}\right\}$, since Stirling formula ensures:

$$
\left(4 \frac{r}{t} C_{r}^{\frac{r}{t}}\right)^{\frac{1}{r}} \sim_{r \rightarrow \infty}\left(4 \frac{r}{t} \frac{\sqrt{(2 \pi r)}}{\sqrt{2 \pi \frac{r}{t}} \sqrt{2 \pi\left(1-\frac{1}{t}\right) r}}\right)^{\frac{1}{r}} \frac{\frac{r}{e}}{\left(\frac{r}{e t}\right)^{\frac{1}{t}}\left(\left(1-\frac{1}{t}\right) \frac{r}{e}\right)^{1-\frac{1}{t}}} \sim_{r \rightarrow \infty} t^{\frac{1}{t}}\left(1-\frac{1}{t}\right)^{\frac{1}{t}-1} .
$$

The estimate is valid for any level $k$ so that for all parameter $t \geq 2$ :

$$
c_{k} \leq \max \left\{c_{k+1}^{1-\frac{1}{t}}, t^{\frac{1}{t}}\left(1-\frac{1}{t}\right)^{\frac{1}{t}-1}\right\} .
$$

In particular, this shows the sequence $\left(c_{k}\right)_{k}$ increases (note $t^{\frac{1}{t}}\left(1-\frac{1}{t}\right)^{\frac{1}{t}-1} \rightarrow 1$ for $t \rightarrow \infty$ ). Moreover the sequence is bounded by 2 (the groups are quotients of $\mathbb{Z} / 2 \mathbb{Z} * \mathbb{Z} / 3 \mathbb{Z}$ ), hence admits a limit $c_{\infty}$, which satisfies by continuity:

$$
c_{\infty} \leq \max \left\{c_{\infty}^{1-\frac{1}{t}}, t^{\frac{1}{t}}\left(1-\frac{1}{t}\right)^{\frac{1}{t}-1}\right\}
$$

for any parameter $t \geq 2$, which is impossible unless $c_{\infty}=1$ (otherwise take $t$ large enough). This shows subexponential growth of the groups $H_{\sigma^{k}} \bar{d}$.

Remark 6.7. When the tree has bounded valency, set $f(r)=\max \left\{b_{k}(r) \mid k \in \mathbb{N}\right\}$ the estimate (6.6) can be made homogeneous on $d_{k} \leq D$. This together with estimate (6.7) applied for a parameter $t$ of the form $t=\frac{\bar{K}}{\log (r)}$ raises inequality:

$$
f(r) \leq K\left(\sum_{r_{1}+\cdots+r_{D} \leq\left(1-\frac{K}{\log (r)}\right) r} \prod_{i=1}^{D} f\left(r_{i}\right)\right)+K C_{r}^{\frac{K}{\log (r)}} .
$$

A computation due to Erschler (Lemma 6.4 in [Ers]) gives the explicit upper bound on the growth:

$$
b_{0}(r) \leq f(r) \leq \exp \left(\frac{K \log (\log (r)) r}{\log (r)}\right)
$$




\section{GROUPS OF NON UNIFORM GROWTH}

7.1. A Theorem of Wilson. The first examples of groups of non uniform exponential growth have been constructed by Wilson in [Wil1]. The following Theorem from [Wil2] is a generalization.

Theorem 7.1 (Wilson [Wil2]). Let $k$ be a positive integer and $\chi_{k}$ a class of groups with the two properties:

(1) each group $G$ in $\chi_{k}$ is perfect (that is $G=[G, G]$ ) and can be generated by $k$ involutions;

(2) each group $G$ in $\chi_{k}$ is isomorphic to a permutational wreath product $G_{1} \prec \mathcal{A}_{d}$ with $G_{1} \in \chi_{k}$ and $d \geq 29$.

Then each group $G$ in $\chi_{k}$ contains two sequences of elements $\left(a^{(n)}\right),\left(b^{(n)}\right)$ such that:

(a) $\left(a^{(n)}\right)^{2}=\left(b^{(n)}\right)^{3}=1$ and $\left\langle a^{(n)}, b^{(n)}\right\rangle=G$ for each $n$ and,

(b) $h_{\left\{a^{(n)}, b^{(n)}\right\}}(G) \rightarrow 1$ as $n \rightarrow \infty$.

In section 4. of [Wil2], Wilson constructs subgroups of $A u t^{e}\left(T_{\bar{d}}\right)$ for unbounded sequences $\bar{d}=\left(d_{i}\right)_{i}$ in the classes $\chi_{k}$. Unboundedness of the sequence permits to construct such groups with a subgroup isomorphic to the free group $\mathbb{F}_{2}$ on two generators. This ensures exponential growth, but prevents amenability.

In the next section groups in the class $\chi_{k}$ are constructed similarly but acting on bounded valency rooted tree. The Main Theorem 3.1 will apply to show amenability. Exponential growth is due to the presence of free semigroups. Note however that in [Wil1] Wilson constructs groups of automorphism of a regular (in particular bounded valency) rooted tree which have non uniform growth and contain a free group.

7.2. Amenable groups of non uniform growth. Let $\bar{d}=\left(d_{i}\right)_{i}$ be a bounded sequence of integers $5 \leq d_{i} \leq D$, define a subgroup of the group $\bar{H}$ (constructed in section 2.3) as $\bar{A}<\overline{\bar{H}}=S_{d_{1}}^{d_{0}-1} \times S_{d_{2}}^{d_{1}-1} \times \ldots$ where $\bar{A}=\mathcal{A}_{d_{1}} \times \mathcal{A}_{d_{2}} \times \ldots$ as an abstract group and each group $\mathcal{A}_{d_{k}}$ is acting as a rooted automorphism on $T_{1_{k-1} 2}$; this is best understood by Figure 4 .

Now for each integer $d$ in $\{5, \ldots, D\}$, denote $E_{d}=\left\{i \geq 1 \mid d_{i}=d\right\}$. There is a diagonal injection:

$$
j_{d}: \mathcal{A}_{d} \hookrightarrow \prod_{i \in E_{d}} \mathcal{A}_{d_{i}}<\bar{A},
$$

and the diagonal product of those injections:

$$
j: A_{\bar{d}}=\prod_{d=5}^{D} \mathcal{A}_{d} \hookrightarrow \bar{A} .
$$

To ease notation the image subgroup of $A_{\bar{d}}$ is still denoted $A_{\bar{d}}$. It is a finite saturated subgroup of $\bar{A}$. The subgroup of $A u t^{e}\left(T_{\bar{d}}\right)$ generated by alternate rooted automorphisms $\mathcal{A}_{d_{0}}$ and $A_{\bar{d}}$ is denoted $G_{0}=G\left(\mathcal{A}_{d_{0}}, A_{\bar{d}}\right)<A u t^{e}\left(T_{\bar{d}}\right)$. Note that when $\bar{d}=\sigma \bar{d}$ is a constant sequence, the group $G\left(\mathcal{A}_{d}, A_{\bar{d}}\right)$ is generated by a finite automaton. 


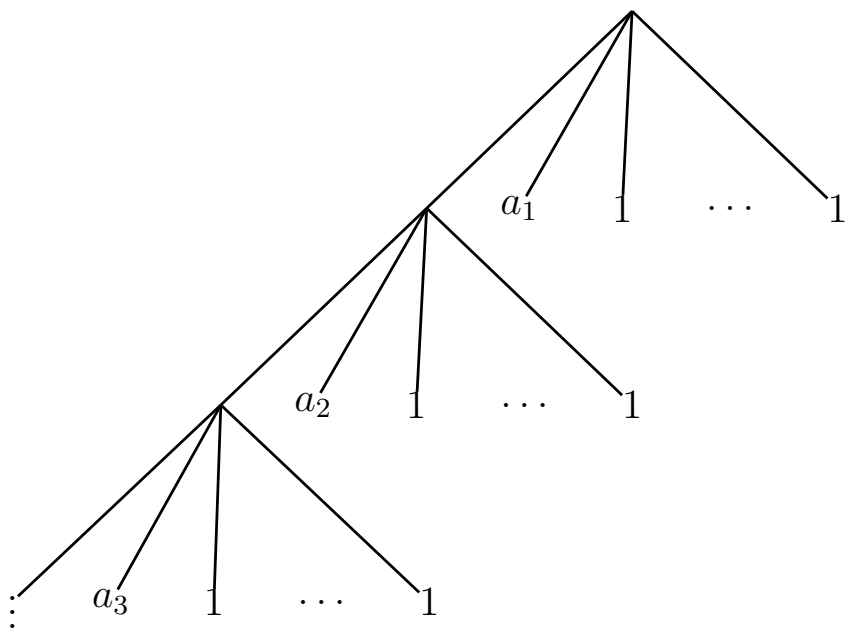

Figure 4. The group $\bar{A}$.

Proposition 7.2. Let $\bar{d}=\left(d_{i}\right)_{i}$ a bounded sequence of integers $29 \leq d_{i} \leq D$, the group $G_{0}=G\left(\mathcal{A}_{d_{0}}, A_{\bar{d}}\right)$ belongs to the class $\chi_{k}$ where $k$ depends only on $D$.

Proof. Show this Proposition simultaneously for all groups $G_{i}=G\left(\mathcal{A}_{d_{i}}, A_{\sigma^{i} \bar{d}}\right)<$ $A u t^{e}\left(T_{\sigma^{i} \bar{d}}\right)$. The group $G_{i}$ is perfect because generated by copies of the groups $\mathcal{A}_{d_{i}}$, $\mathcal{A}_{d}, d \in\{5, \ldots, D\}$ which are perfect (even simple). Moreover, those groups (hence $G_{i}$ ) are generated by double transpositions, in particular by involutions the number of which depends only on $D$, so that the condition (1) of definition of groups in the class $\chi_{k}$ is satisfied for some $k$ depending only on $D$.

To check condition (2), note first that the injection in the wreath product (2.1) has image in:

$$
G_{i}=G\left(\mathcal{A}_{d_{i}}, A_{\sigma^{i} \bar{d}}\right) \hookrightarrow G\left(\mathcal{A}_{d_{i+1}}, A_{\sigma^{i+1}}\right) \curlywedge \mathcal{A}_{d_{i}}=G_{i+1} \prec \mathcal{A}_{d_{i}} .
$$

This is clear for the generators in $\mathcal{A}_{d_{i}}$ and the generators $b$ in $A_{\sigma^{i} \bar{d}}$ have image $b=\left(b^{\prime}, a, 1, \ldots, 1\right)$ where $a$ belongs to $\mathcal{A}_{d_{i+1}}$ and $b^{\prime}$ to $A_{\sigma^{i+1}} \bar{d}$ by construction. Now remains to prove this injection is onto hence an isomorphism.

Given any two elements $a_{1}, a_{2}$ in $\mathcal{A}_{d_{i+1}}$ there exists $b_{1}=\left(b_{1}^{\prime}, a_{1}, 1, \ldots, 1\right)$ and $b_{2}=\left(b_{2}^{\prime}, a_{2}, 1, \ldots, 1\right)$ in $A_{\sigma^{i} \bar{d}}$. Moreover the double transposition $\sigma=(13)(45)$ belongs to $\mathcal{A}_{d_{i}}$, so that $G_{i}$ contains $b_{2}^{\sigma}=\sigma b_{2} \sigma^{-1}=\left(1, a_{2}, b_{2}^{\prime}, 1, \ldots, 1\right)$, hence $\left[b_{1}, b_{2}^{\sigma}\right]=$ $\left(1,\left[a_{1}, a_{2}\right], 1, \ldots, 1\right)$ and then $1 \times \mathcal{A}_{d_{i+1}} \times \cdots \times 1$ by perfection. Similarly given any two $b_{1}^{\prime}, b_{2}^{\prime}$ in $A_{\sigma^{i+1}}$, the group $G_{i}$ contains $\left[b_{1}, b_{2}^{\tau}\right]=\left(\left[b_{1}^{\prime}, b_{2}^{\prime}\right], 1, \ldots, 1\right)$ where $\tau=(23)(45)$, hence $A_{\sigma^{i+1} \bar{d}} \times 1 \times \cdots \times 1$. Since $\mathcal{A}_{d_{i}}$ acts transitively by conjugation on the coordinates, this proves injection (7.1) is onto.

Proposition 7.3. Let $\bar{d}=\left(d_{i}\right)_{i}$ a bounded sequence of integers $5 \leq d_{i} \leq D$, the group $G_{0}=G\left(\mathcal{A}_{d_{0}}, A_{\bar{d}}\right)$ has exponential growth.

Proof. Each group $\mathcal{A}_{d}$ contains the double transpositions $u=(12)(34)$ and $v=$ (12)(35). Moreover each of the groups $A_{\sigma^{i} \bar{d}} \simeq \mathcal{A}_{d_{i}} \times \mathcal{A}_{d_{i+1}} \times \ldots$ contains the diagonal elements $\bar{u}=(u, u, u, \ldots)$ and $\bar{v}=(v, v, v, \ldots)$. The following Lemma due to 
Bartholdi (Proposition 2.3 in $\left[\right.$ Bar]) ensures that $\langle\bar{u} u, \bar{v} v\rangle \simeq \mathbb{S}_{2}$ is a free semigroup. More precisely:

Lemma 7.4 (Bartholdi $[\mathrm{Bar}]$ ). The quotient semigroup

$$
\langle\bar{u} u, \bar{u} v, \bar{v} u, \bar{v} v\rangle /(\bar{u} u=\bar{u} v, \bar{v} u=\bar{v} v) \simeq \mathbb{S}_{2}
$$

is freely generated by $\{\bar{u} u, \bar{v} v\}$.

This ensures exponential growth of the group $G_{0}$.

Corollary 7.5 (Theorem 1.1). The groups $G\left(\mathcal{A}_{d_{0}}, A_{\bar{d}}\right)$ associated to sequences $\bar{d}=$ $\left(d_{i}\right)_{i}$ of integers $29 \leq d_{i} \leq D$ are (uncountably many pairwise non isomorphic) amenable groups of non uniform exponential growth.

Proof. This follows from the Main Theorem 3.1, Wilson's Theorem 7.1, Proposition 7.2 and Proposition 7.3. The bracketted part follows from Corollary 8.2.

7.3. Convergence of the Cayley graphs. This section is devoted to give another proof of some part of Wilson Theorem 7.1. Namely the convergence to 1 of the exponential growth rate of the generating sets $\left\{a^{(n)}, b^{(n)}\right\}$ can be understood as the convergence of the associated Cayley graphs of the group to the Cayley graph of a group $H_{\bar{d}}$ of intermediate growth introduced in section 6 .

More precisely, let $G=G_{0}$ belong to some class $\chi_{k}$, then by definition of the class there exists a sequence of groups $G_{i}$ in $\chi_{k}$ and integers $d_{i} \geq 29$ such that $G_{i} \simeq G_{i+1}$ 2 $\mathcal{A}_{d_{i}}$. The Theorem 7.1 of Wilson ensures in particular that for each integer $i$ there exists a generating pair of elements $\left\langle a_{i}^{(0)}, b_{i}^{(0)}\right\rangle=G_{i}$ such that $\left(a_{i}^{(0)}\right)^{2}=\left(b_{i}^{(0)}\right)^{3}=1$. Out of this first generating pair, Wilson constructs a sequence of generating pairs for $G_{i}$, defined inductively as (also see Figure 5 and compare with Figure 3):

$$
\begin{aligned}
& a_{i}^{(n+1)}=\left(a_{i+1}^{(n)}, 1, \ldots, 1\right) x_{d_{i}}, \\
& b_{i}^{(n+1)}=\left(1, b_{i+1}^{(n+1)}, 1, \ldots, 1\right) y_{d_{i}} .
\end{aligned}
$$

The fact that $a_{i}^{(n)}$ and $b_{i}^{(n)}$ have order 2 and 3 and that they generate $G_{i}$ is a direct consequence of the properties of the generating pairs $x_{d_{i}}, y_{d_{i}}$ of the alternate group $\mathcal{A}_{d_{i}}$ (see Proposition 6.1).

Definition 7.6 (Distance between Cayley graphs). Let $(\Gamma, S)$ and $(\Delta, T)$ be two groups with generating sets, denote $B_{\Gamma, S}(R)$ the restriction of the Cayley graph of $\Gamma$ relatively to the generating set $S$ to vertices at distance less than $R$ of the neutral element (for the word distance in $S$ ). The distance between $(\Gamma, S)$ and $(\Delta, T)$ is defined as:

$$
d((\Gamma, S),(\Delta, T))=\inf \left\{\frac{1}{R} \mid B_{\Gamma, S}(R) \sim_{G} B_{\Delta, T}(R)\right\},
$$

where $G r_{1} \sim_{G} G r_{2}$ if $G r_{1}$ and $G r_{2}$ are isometric as colored graphs.

Non uniform growth of the group $G_{0}$ comes from the two next propositions, since intermediate growth of $H_{\bar{d}}$ implies $h_{\left\{x_{\bar{d}}, y_{\bar{d}}\right\}}\left(H_{\bar{d}}\right)=1$. 

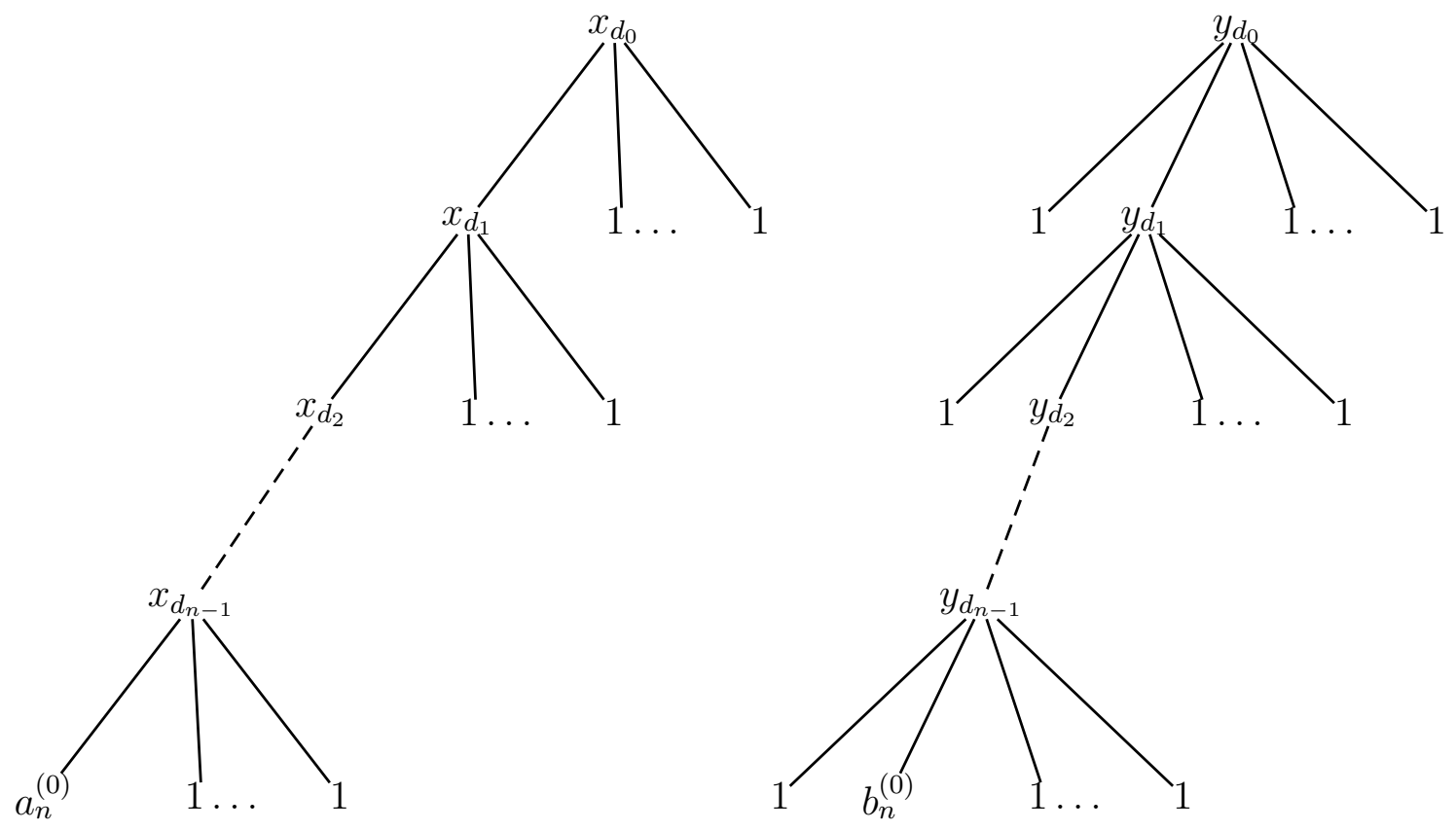

FiguRE 5. Portraits of the elements $a_{0}^{(n)}$ and $b_{0}^{(n)}$.

Proposition 7.7. With the notations above:

$$
d\left(\left(G_{0},\left\{a_{0}^{(n)}, b_{0}^{(n)}\right\}\right),\left(H_{\bar{d}},\left\{x_{\bar{d}}, y_{\bar{d}}\right\}\right)\right) \underset{n \rightarrow+\infty}{\longrightarrow} 0 .
$$

Note that this Proposition is true independently of the amenability or not of the group $G_{0}$ in a class $\chi_{k}$. In particular, such a convergence is also true for the non amenable groups constructed by Wilson in [Wil1], [Wil2].

Proposition 7.8. If $d\left(\left(\Gamma, S_{n}\right),(\Delta, T)\right) \rightarrow 0$, then:

$$
\limsup _{n \rightarrow \infty} h_{S_{n}}(\Gamma) \leq h_{T}(\Delta) .
$$

Proof of Proposition 7.8. Given a positive $\varepsilon$ the definition of $h_{T}(\Delta)$ ensures that for $R \geq R_{0}$ large enough the ball $B_{\Delta, T}(R)$ has size $\# B_{\Delta, T}(R) \leq\left(h_{T}(\Delta)+\varepsilon\right)^{R}$. Now the convergence of Cayley graphs shows that for $n \geq N$ large enough $\# B_{\Gamma, S_{n}}(R) \leq$ $\left(h_{T}(\Delta)+\varepsilon\right)^{R}$, and by subadditivity $\# B_{\Gamma, S_{n}}(k R) \leq \# B_{\Gamma, S_{n}}(R)^{k} \leq\left(h_{T}(\Delta)+\varepsilon\right)^{k R}$, so that:

$$
h_{S_{n}}(\Gamma)=\lim \sqrt[k R]{\# B_{\Gamma, S_{n}}(k R)} \leq h_{T}(\Delta)+\varepsilon
$$

which was required.

The proof of Proposition 7.7 uses the following:

Lemma 7.9 (of contraction). If $\hat{x}=(u, 1, \ldots, 1) x_{d}$ and $\hat{y}=(1, v, 1, \ldots, 1) y_{d}$ are as in Proposition 6.1, then for elements $g=\left(g_{1}, \ldots, g_{d}\right) \sigma$ in the wreath product isomorphism $\langle\hat{x}, \hat{y}\rangle \simeq\langle u, v\rangle\left\langle\mathcal{A}_{d}\right.$, one has for each coordinate $t$ :

$$
\left|g_{t}\right|_{\{u, v\}} \leq \frac{1}{2}\left(|g|_{\{\hat{x}, \hat{y}\}}+1\right),
$$


where $|\cdot|_{S}$ denotes the word norm associated to the generating set $S$ (inverses of elements of $S$ have length 1).

Proof. It is sufficient to check that $\hat{x} \hat{y}^{\varepsilon}=\left(u, 1, \ldots, v^{\varepsilon}, 1, \ldots, 1\right) x_{d} y_{d}$ with $v^{\varepsilon}$ on coordinate $x_{d}(2) \neq 1$.

Proof of Proposition 7.7. Introduce other relations depending on integer $l \geq 1$ on groups with generating sets: $(\Gamma, S) \sim_{l}(\Delta, T)$ if for every free word $w$ of length less than $l$ in $S$ (elements and inverses) one has $w(S)=i d_{\Gamma}$ if and only if $w(T)=i d_{\Delta}$ (for $l=1$ the relation $\sim_{1}$ just means $S \cup S^{-1}$ and $T \cup T^{-1}$ have the same size). If the relation $(\Gamma, S) \sim_{2 l+1}(\Delta, T)$ is satisfied then $d((\Gamma, S),(\Delta, T)) \leq \frac{1}{l}$ because to describe $B_{\Gamma, S}(R)$ it is sufficient to know when $g^{\prime} g^{-1}=s$ for every $g, g^{\prime}$ in $B_{\Gamma, S}(R)$ and $s$ in $S \cup S^{-1}$.

To ease notations set $S_{i}^{(n)}=\left\{a_{i}^{(n)}, b_{i}^{(n)}\right\}$ and $T_{i}=\left\{x_{\sigma^{i} \bar{d}}, y_{\sigma^{i} \bar{d}}\right\}$. It is sufficient to show for all integers $i$ : $\left(G_{i}, S_{i}^{(n)}\right) \sim_{l_{n}}\left(H_{\sigma^{i} \bar{d}}, T_{i}\right)$ with a sequence $l_{n} \rightarrow \infty$. Proceed by induction on $n$, using:

$$
\begin{aligned}
& w\left(S_{i}^{(n+1)}\right)=\left(w_{1}\left(S_{i+1}^{(n)}\right), \ldots, w_{d_{i}}\left(S_{i+1}^{(n)}\right)\right) w\left(x_{d_{i}}, y_{d_{i}}\right), \\
& w\left(T_{i}\right)=\left(w_{1}\left(T_{i+1}\right), \ldots, w_{d_{i}}\left(T_{i+1}\right)\right) w\left(x_{d_{i}}, y_{d_{i}}\right),
\end{aligned}
$$

where for each coordinate $t$ the elements $w_{t}\left(S_{i+1}^{(n)}\right)$ and $w_{t}\left(T_{i+1}\right)$ involve the same word $w_{t}$ because the permutations on the first level are the same for generators in $S_{i}^{(n+1)}$ or in $T_{i}$ (namely $x_{d_{i}}$ and $y_{d_{i}}$ ). Lemma 7.9 ensures that $\left|w_{t}\left(T_{i+1}\right)\right| \leq \frac{1}{2}\left(\left|w\left(T_{i}\right)\right|+1\right.$ ) (and $\left|w_{t}\left(S_{i+1}^{(n)}\right)\right| \leq \frac{1}{2}\left(\left|w\left(S_{i}^{(n+1)}\right)\right|+1\right)$ ) so that if $w$ has length less than $l_{n+1}=2 l_{n}-1$ one has $w\left(S_{i}^{(n+1)}\right)=i d_{G_{i}}$ if and only if $w\left(T_{i}\right)=i d_{H_{\sigma^{i} \bar{d}}}$. The result follows since the sequence $\left(l_{n}\right)$ starts with $l_{0}=1$ and $l_{1}=2$.

Corollary 7.10 (of Proposition 7.7). The group $H_{\bar{d}}$ of intermediate growth is not finitely presented.

Proof. Assume the contrary $H_{\bar{d}}=\left\langle x_{\bar{d}}, y_{\bar{d}} \mid r_{1}, \ldots, r_{k}\right\rangle$. Let $R$ be bigger than the maximal length of the relations $r_{1}, \ldots, r_{k}$, and $n$ large enough so that:

$$
d\left(\left(G_{0},\left\{a_{0}^{(n)}, b_{0}^{(n)}\right\}\right),\left(H_{\bar{d}},\left\{x_{\bar{d}}, y_{\bar{d}}\right\}\right)\right) \leq \frac{1}{R} .
$$

Then the automorphisms $a_{0}^{(n)}$ and $b_{0}^{(n)}$ satisfy all relation $r_{1}, \ldots, r_{k}$. In particular, $G_{0}$ is a quotient of $H_{\bar{d}}$ hence has subexponential growth. This contradicts Proposition 7.3.

\section{NON SUBEXPONENTIAL AMENABILITY}

8.1. Description of normal subgroups. The normal subgroups of finite index of groups in a class $\chi_{k}$ are completely described by the:

Proposition 8.1 (Neumann [Neu]). Let $\left(G_{i}\right)_{i \in \mathbb{N}}$ be a sequence of finitely generated perfect groups such that for each $i$ there exists an integer $d_{i} \geq 5$ such that $G_{i} \simeq$ 
$G_{i+1} \prec \mathcal{A}_{d_{i}}$. Consider the isomorphisms:

$$
G_{0} \simeq G_{i} \curlywedge \mathcal{A}_{d_{i-1}} \prec \cdots \succ \mathcal{A}_{d_{0}} \simeq \underbrace{\left(G_{i} \times \cdots \times G_{i}\right)}_{d_{0} \ldots d_{i-1} \text { times }} \rtimes A u t^{e}\left(T_{d_{0} \ldots d_{i-1}}\right),
$$

then the subgroups $K_{i}=\left(G_{i} \times \cdots \times G_{i}\right)$ for $i \in \mathbb{N}$ are the only normal subgroups of $G_{0}$ of finite index. Moreover if one (hence all) of the groups $G_{i}$ is residually finite, then $\left(K_{i}\right)_{i \in \mathbb{N}}$ are the only non trivial normal subgroups of $G_{0}$; in particular $G_{0}$ is just infinite.

Proposition 8.1 (as well as Lemma 8.3) is a slight generalization of Theorem 5.1. in [Neu]. The proof is given here for the sake of completeness and to avoid the reader multiple references and notations. The second part is also similar to Theorem 4. in [Gri2]. Note that all examples in this paper are groups of automorphism of a rooted tree. In particular they satisfy the assumption of residual finiteness.

Corollary 8.2. Two groups $G_{0}$ and $H_{0}$ satisfying the hypothesis of Proposition 8.1 (in particular groups in a class $\chi_{k}$ ) for two different sequences of integers $\left(d_{i}\right)_{i}$ and $\left(e_{i}\right)_{i}$ are non isomorphic.

Proof. The index of $K_{i}$ in $G_{0}$ has value:

$\left[G_{0}: K_{i}\right]=\# A u t^{e}\left(T_{d_{0} \ldots d_{i-1}}\right)=\#\left(\mathcal{A}_{d_{i-1}} \prec \cdots \prec \mathcal{A}_{d_{0}}\right)=a\left(d_{i-1}\right)^{d_{i-2} \ldots d_{0}} \ldots a\left(d_{1}\right)^{d_{0}} a\left(d_{0}\right)$, where $a(d)=\frac{d !}{2}=\# \mathcal{A}_{d}$. In particular the sequence of index of subgroups $\left(\left[G_{0}: K_{i}\right]\right)_{i}$ is an isomorphism invariant from which the sequence $\left(d_{i}\right)_{i}$ can be recovered.

Lemma 8.3. Under the hypothesis of Proposition 8.1, the only normal subgroups of $G_{0}$ containing $K_{m}$ are $K_{0}, K_{1}, \ldots, K_{m}$.

The proof of this lemma will use the:

Fact 8.4. Given a finite group $\Gamma$, assume $\Delta \triangleleft \Gamma$ is a minimal normal subgroup (minimal means the only normal subgroup of $\Gamma$ strictly contained in $\Delta$ is trivial) and that the centralizer $\operatorname{Cent}_{\Gamma}(\Delta)$ of $\Delta$ is trivial. Then $\Delta$ is the unique minimal normal subgroup of $\Gamma$.

Proof. Assume $\Delta^{\prime}$ is another such subgroup, then $\Delta \cap \Delta^{\prime}$ is trivial by minimality. In particular, for every $\delta \in \Delta$ and $\delta^{\prime} \in \Delta^{\prime}$ the commutator $\Delta \cap \Delta^{\prime} \ni\left[\delta, \delta^{\prime}\right]=1$, which ensures $\Delta^{\prime} \subset \operatorname{Cent}_{\Gamma}(\Delta)=\{1\}$, contradiction.

Proof of Lemma 8.3. By induction on $m$ and using Fact 8.4, it is sufficient to prove that:

$$
\mathcal{A}_{d_{m-1}}^{(1 \ldots 1)} \times \cdots \times \mathcal{A}_{d_{m-1}}^{\left(d_{0} \ldots d_{m-2}\right)} \simeq K_{m-1} / K_{m} \triangleleft G_{0} / K_{m} \simeq \operatorname{Aut}^{e}\left(T_{d_{0} \ldots d_{m-1}}\right)
$$

is minimal and has trivial centralizer, which shows $K_{m-1}$ is the only minimal subgroup of $G_{0}$ containing $K_{m}$.

Let $U$ a non trivial subgroup normal in $G_{0} / K_{m}$ and included in $K_{m-1} / K_{m}$. Then $1 \neq y \in U$ can be written $y=\left(y_{1 \ldots 1}, \ldots, y_{d_{0} \ldots d_{m-1}}\right)$ in the wreath product $G_{0} / K_{m} \simeq$ $\mathcal{A}_{d_{m-1}}$ 2 Aut ${ }^{e}\left(T_{d_{0} \ldots d_{m-2}}\right)$, with some coordinate $1 \neq y_{v} \in \mathcal{A}_{d_{m-1}}^{(v)}$. By simplicity, the normal closure of $y_{v}$ is the full alternate group $\left\langle y_{v}\right\rangle_{\mathcal{A}_{d_{m-1}}}=\mathcal{A}_{d_{m-1}}$. Moreover 
the group $A u t^{e}\left(T_{d_{0} \ldots d_{m-2}}\right)$ acts by conjugation transitively on the coordinates, so that $U>\langle y\rangle_{G_{0} / K_{m}}=\mathcal{A}_{d_{m-1}}^{(1 \ldots 1)} \times \cdots \times \mathcal{A}_{d_{m-1}}^{\left(d_{0} \ldots d_{m-2}\right)}=K_{m-1} / K_{m}$, proving minimality. Transitivity also shows that the centralizer $\operatorname{Cent}_{G_{0} / K_{m}}\left(K_{m-1} / K_{m}\right)$ is included in $S t_{m-1}\left(G_{0} / K_{m}\right)=K_{m-1} / K_{m}$, which has trivial center, hence the centralizer is trivial.

Proof of Proposition 8.1. Let $X$ be a finite group and $f: G_{0} \rightarrow X$ a homomorphism. Restricting to factors of the subgroups $K_{m}=G_{m}^{(1 \ldots 1)} \times \cdots \times G_{m}^{(v)} \times \cdots \times G_{m}^{\left(d_{0} \ldots d_{m-1}\right)}$, it appears that for $m$ large enough there exists $v \neq v^{\prime}$ such that the associated factors have the same image $f\left(G_{m}^{(v)}\right)=f\left(G_{m}^{\left(v^{\prime}\right)}\right)=Y$, which must be abelian because $\left[G_{m}^{(v)}, G_{m}^{\left(v^{\prime}\right)}\right]=1$, hence $Y=\{1\}$ because $G_{m}^{(v)} \simeq G_{m}$ is perfect. This shows $G_{m}^{(v)} \subset$ $\operatorname{Ker}(f)$.

Moreover for each coordinate $v^{\prime}$ there exists $\varphi \in A u t^{e}\left(T_{d_{0} \ldots d_{m-1}}\right)$ such that $\varphi(v)=$ $v^{\prime}$, so that $\varphi G_{m}^{(v)} \varphi^{-1}=G_{m}^{\left(v^{\prime}\right)} \subset \operatorname{Ker}(f)$ and consequently $K_{m}$ lies in the kernel of $f$. Applying Lemma 8.3 shows $\operatorname{Ker}(f)=K_{i}$ for some $i \leq m$, which proves the first part.

Now assume $G_{0}$ is residually finite, and $N \triangleleft G_{0}$ is an arbitrary normal subgroup. The description of the first part ensures that $\cap_{m \geq 0} K_{m}=\{1\}$, and as the sequence of subgroups $\left(K_{m}\right)_{m}$ is strictly decreasing there exists an integer $n$ such that $N \leq K_{n}$ and $N \not \leq K_{n+1}$. To get the second part, it is sufficient to prove $N \geq K_{n+1}$ since the first part will force $K_{n}=N$.

Consider $x \in N \backslash K_{n+1}$ and its image $x=\left(x_{1 \ldots 1}, \ldots x_{d_{0} \ldots d_{n-1}}\right)_{n}$ in the factor decomposition of $K_{n}$. There exists $v$ such that:

$$
x_{v}=\left(x_{v 1}, \ldots, x_{v d_{n}}\right) \sigma_{v} \in G_{n}^{(v)} \simeq G_{n+1} \prec \mathcal{A}_{d_{n}},
$$

with a non trivial permutation $\sigma_{v}$, and in particular there are $s \neq t$ in $\left\{1, \ldots d_{n}\right\}$ with $\sigma_{v}(s)=t$. Now given any two elements $\xi, \eta$ in $G_{n+1}$, define $f, g$ in $K_{n}=$ $\left(G_{n} \times \cdots \times G_{n}\right)$ as:

$$
\begin{aligned}
& f=\left(1, \ldots, 1, f_{v}, 1 \ldots, 1\right)_{n}, \quad f_{v}=(1, \ldots, 1, \xi, 1, \ldots, 1) \in G_{n+1} \prec \mathcal{A}_{d_{n}}, \\
& g=\left(1, \ldots, 1, g_{v}, 1 \ldots, 1\right)_{n}, \quad g_{v}=(1, \ldots, 1, \eta, 1, \ldots, 1) \in G_{n+1} \prec \mathcal{A}_{d_{n}},
\end{aligned}
$$

with $\xi, \eta$ on coordinate $s$. The normal subgroup $N$ contains the commutator $[f, x]=$ $f x f^{-1} x^{-1}=\left(1, \ldots, 1,\left[f_{v}, x_{v}\right], 1, \ldots, 1\right)_{n}$, where:

$$
\begin{aligned}
{\left[f_{v}, x_{v}\right] } & =(1, \ldots, \xi, \ldots, 1)\left(x_{v 1}, \ldots, x_{v d_{n}}\right) \sigma_{v}\left(1, \ldots, \xi^{-1}, \ldots, 1\right) \sigma_{v}^{-1}\left(x_{v 1}^{-1}, \ldots, x_{v d_{n}}^{-1}\right) \\
& =\left(1, \ldots, 1, \xi, 1, \ldots, 1, x_{v t} \xi^{-1} x_{v t}^{-1}, 1, \ldots, 1\right)
\end{aligned}
$$

with $\xi$ in coordinate $s$ and $x_{v t} \xi^{-1} x_{v t}^{-1}$ in coordinate $t$. Taking another commutator, the subgroup $N$ contains $[g,[f, x]]=\left(1, \ldots, 1,\left[g_{v},\left[f_{v}, x_{v}\right]\right], 1, \ldots, 1\right)_{n}$ with:

$$
\left[g_{v},\left[f_{v}, x_{v}\right]\right]=(1, \ldots, 1,[\eta, \xi], 1, \ldots, 1),
$$

and this for $\xi, \eta$ in $G_{n+1}$ arbitrary, which can be rewritten:

$$
N \ni(1, \ldots, 1,[\eta, \xi], 1, \ldots, 1)_{n+1} \in G_{n+1} \prec A u t^{e}\left(T_{d_{0} \ldots d_{n}}\right),
$$

with $[\eta, \xi]$ in position $v s$. As this group is perfect, the subgroup $N$ contains $1 \times$ $\cdots \times G_{n+1}^{(v s)} \times \cdots \times 1$. The transitivity of the action of $A u t^{e}\left(T_{d_{0} \ldots d_{n}}\right)$ on level $n+1$ by conjugation ensures that $N$ contains $\left(G_{n+1} \times \cdots \times G_{n+1}\right)=K_{n+1}$ as required. 
8.2. Non subexponential amenability. Denote $S G_{0}$ (respectively $E G_{0}$ ) the class of groups such that all finitely generated subgroups have subexponential growth (respectively are abelian). Assume that for an ordinal $\alpha>0$ the classes $S G_{\beta}$ and $E G_{\beta}$ are defined for every ordinal $\beta<\alpha$. When $\alpha$ is a limit ordinal, set $S G_{\alpha}=\cup_{\beta<\alpha} S G_{\beta}$ (respectively $E G_{\alpha}=\cup_{\beta<\alpha} E G_{\beta}$ ). When $\alpha$ is a successor ordinal, define $S G_{\alpha}$ (respectively $E G_{\alpha}$ ) to be the class of groups that can be obtained from groups in the class $S G_{\alpha-1}$ (respectively $E G_{\alpha-1}$ ) either by taking direct limits, or by taking extension by a group from the class $S G_{0}$ (respectively $E G_{0}$ ).

Each class $S G_{\alpha}$ (respectively $E G_{\alpha}$ ) is closed under taking quotients and subgroups. Moreover, the class $S G=\cup_{\alpha} S G_{\alpha}$ (respectively $E G=\cup_{\alpha} E G_{\alpha}$ ) where the union runs over all ordinals $\alpha$, is the smallest class of groups containing $S G_{0}$ (respectively $E G_{0}$ ) which is closed under the operations of taking subgroups, quotients, extensions and direct limits. As these operations preserve amenability, which is satisfied in $S G_{0}$ (respectively $E G_{0}$ ), the class $S G$ (respectively $E G$ ) is called class of subexponentially (respectively elementary) amenable groups.

This construction of classes of groups is detailled in [Osi1]. It is obvious that $E G_{\alpha}$ is a subclass of $S G_{\alpha}$ for each ordinal $\alpha$ and that the class $S G$ contains the class $E G$. This inclusion is strict (see [Gri1]) and the Basilica group introduced in [GZ] was the first example of an amenable group out of $S G$. Osin has shown in [Osi2] that the class $E G$ contains no group of non uniform growth. In particular, groups in the class $\chi$ such as the groups $G\left(\mathcal{A}_{d_{0}}, A_{\bar{d}}\right)$ of non uniform exponential growth introduced in section 7.2 are not in $E G$. The following Proposition shows these groups are not even in $S G$, providing uncountably many pairwise non isomorphic examples of amenable groups outside $S G$.

Proposition 8.5. Consider a residually finite group $G$ belonging to a class $\chi_{k}$ (see section 7), then one of the two following holds:

1) either $G$ belongs to the class $S G_{0}$ of groups of subexponential growth,

2 ) or $G$ does not belong to the class $S G$ of subexponentially amenable groups.

In particular, residually finite groups of exponential growth in a class $\chi_{k}$ are not in $S G$.

Recall an elementary property of ordinals:

Fact 8.6 (Theorem $7.3(5)$ in [Kun]). Let $C$ be a non empty set of ordinals, then there exists $x \in C$ such that for every $y \in C$, one has $x \leq y$. In other words, $C$ has a minimum.

Proof of Proposition 8.5. The proof is similar to that in [GZ]. Let $G$ a group in a class $\chi_{k}$ having exponential growth, in particular not in the class $S G_{0}$. Denote $G_{i}$ the group in the class $\chi_{k}$ such that $G=G_{0} \simeq G_{i} \curlywedge \mathcal{A}_{d_{i-1}} \imath \cdots \curlywedge \mathcal{A}_{d_{0}}$. In particular all groups $G_{i}$ have exponential growth. Assume $G_{0}$ lies in the class $S G$, then all the groups $G_{i}$ (which are subgroups of $G_{0}$ ) lie in $S G$. For each integer $i$ define $\alpha_{i}$ to be the minimal ordinal for which $G_{i}$ belongs to $S G_{\alpha_{i}}$ (exists by Fact 8.6). The family $\left\{\alpha_{i}\right\}_{i \in \mathbb{N}}$ admits a minimum $\alpha_{i_{0}}$. Now the ordinal $\alpha_{i_{0}}$ is not a limit ordinal otherwise $G_{i_{0}}$ would belong to $S G_{\beta}$ for some $\beta<\alpha_{i_{0}}$. Moreover, the group $G_{i_{0}}$ is 
not a direct limit of a strictly increasing infinite sequence of groups because it is finitely generated. This forces the existence of $N$ and $H$ in $S G_{\alpha_{i_{0}}-1}$ such that the sequence $1 \rightarrow N \rightarrow G_{i_{0}} \rightarrow H \rightarrow 1$ is exact. But as $G$ hence $G_{i_{0}}$ is residually finite, Proposition 8.1 implies that $N=G_{i_{0}+m}$ for some integer $m$, so that $\alpha_{i_{0}+m} \leq \alpha_{i_{0}}-1$ which contradicts minimality of $\alpha_{i_{0}}$, proving $G$ is not in $S G$.

\section{REFERENCES}

[Ale1] Aleshin S. V., Finite automata and Burnside problem on periodic groups, Math. Notes 11 (1972), 319-328.

[Ale2] Aleshin S. V., A free group of finite automata, Vestnik Moskov. Univ. Ser. I Mat. mekh. 1983, no. 4, 12-14.

[Bar] Bartholdi L., A Wilson group of non-uniformly exponential growth, C. R. Acad. Sci. 336 (2003), no. 7, 549-554.

[BV] Bartholdi L., Virag Balint, Amenability via random walks, Duke Math. J. 130, no. 1 (2005), 39-56.

[dlH] de la Harpe P., Uniform growth in groups of exponential growth, Geom. Dedicata 95 (2002), 1-17.

[Ers] Erschler A., Boundary behavior for groups of subexponential growth, Annals of Mathematics 160 (2004), 1183-1210.

[EMO] Eskin A., Mozes S., Oh H., Uniform exponential growth for linear groups, Int. Mat. Res. Not. 31 (2002), 1675-1683.

[Gre] Greenleaf F. P., Invariant means on topological groups, New York, Van Nostrand Reinhold Company, 1969 (Van Nostrand mathematical Studies, 16).

[Gri1] Grigorchuk R. I., Degrees of growth of finitely generated groups, and the theory of invariant means, Math. USSR Izvestiya 25 (1985) No 2, 259-300

[Gri2] Grigorchuk R. I., Just Infinite Branch Groups, in New Horizons in pro-p Groups, eds. M. de Sautoy, D. Segal and A. Shalev, Prog. Math., Vol 184, Birkhauser, 2000, 121-179

[GZ] Grigorchuk R. I., Zuk ., On a torsion-free weakly branch group defined by a three state automaton, Internat. J. Algebra Comput., Vol. 12, No. 1 (2002), 1-24.

[Gro1] Gromov M., Groups of polynomial growth and expanding maps, Inst. Hautes Études Sci. Publ. Math. 53 (1981), 53-73.

[Gro2] Gromov M., Structures métriques pour les variétés riemanniennes, Paris: CEDIC 1981.

[Kai] Kaimanovich V. A., "Münchhausen Trick" and Amenability of Self-similar Groups, IJAC 15(5-6) (2005), 907-938.

[Kal] Kallenberg O., Foundations of modern probability, New York NY Springer-Verlag, 2002.

[Kes] Kesten H., Full Banach mean values on countable groups, Math. Scand. 7 (1959), 146-156.

[Kou] Koubi M., Croissance uniforme dans les groupes hyperboliques, Ann. Inst. Fourier 48 (1998), 1441-1453.

[Kun] Kunen K., Set theory: an introduction to independence proofs,Amsterdam New York Oxford , North-Holland 1980.

[Muc] Muchnik R., Amenability of Universal 2-Grigorchuk group eprint arXiv:math/0505572

[Neu] Neumann P. M., Some questions of Edjvet and Pride about infinite groups, Illinois Journal Math. 30 (1986), 301-316.

[Osi1] Osin D. V., Elementary Classes of Groups, Mat. Zametki 72 (2002), 84-93.

[Osi2] Osin D. V., Algebraic entropy of elementary amenable groups, Geom. Dedicata 107 (2004), 133-151.

[PR] Polya G., Read R. C., Combinatorial enumeration of groups, graphs, and chemical compounds, New York NY Springer-Verlag, 1987.

[TW] Tamburini M. C., Wilson J. S., A residual property of certain free product, Math. Z. 186 (1984), 525-530.

[Wil1] Wilson J. S., On exponential growth and uniformly exponential growth for groups, Invent. math. 155 (2004), 287-303. 
[Wil2] Wilson J. S., Further groups that do not have uniformly exponential growth, Journal of Algebra 279 (2004), 292-301.

(J. Brieussel) Université Denis Diderot Paris 7, Institut Mathématique de Jussieu, 175 Rue du Chevaleret, 75013 Paris, France

E-mail address: brieussel@math.jussieu.fr 